\title{
1 Endocardial differentiation in zebrafish occurs during early \\ 2 somitogenesis and is dependent on BMP and etv2 signalling
}

3 Samuel J Capon ${ }^{1}$ and Kelly A Smith ${ }^{1 *}$

$4{ }^{1}$ Division of Genomics of Development and Disease, Institute for Molecular

5 Bioscience, The University of Queensland, Brisbane, Queensland 4072,

6 Australia.

$7 \quad$ * Author for correspondence, email: k.smith@imb.uq.edu.au 


\section{Abstract:}

9 The endocardium and adjacent vascular endothelial network share a number

10 of molecular markers however there are distinct physiological functions of

11 these tissues. What distinguishes these lineages on a molecular level remains

12 an important, unanswered question in cardiovascular biology. We have

13 identified the Gt(SAGFF27C); $T g(4 x U A S$ :egfp) line as a marker of early

14 endocardial development and used this line to examine endocardial

15 differentiation. Our results show that the endocardium emerges from the

16 anterior lateral plate mesoderm at the 8-somite stage (13 hpf). Analysis in a

17 number of loss-of-function models showed that whilst $n k x 2.5$, hand 2 and tal1

18 loss-of-function have no effect on the endocardial progenitor domain, both

19 etv2 loss-of-function and inhibition of BMP signalling reduce the endocardial

20 domain. Furthermore, manipulating BMP signalling alters etv2 expression.

21 Together, these results describe the onset of endocardial molecular identity

22 and suggest a signalling cascade whereby BMP signalling acts upstream of

23 etv2 to direct differentiation of endocardial progenitors. 


\section{Introduction:}

During early stages of embryonic development the heart is comprised of two major tissues: the myocardium and endocardium. To date, much of the research concerning heart development has focused on the myocardium, the beating muscle of the heart, while the endocardium has received relatively little attention. The endocardium is a specialised subset of endothelium that forms the inner lining of the heart. It plays important roles in the development of the trabecular myocardium, contributes to the coronary vasculature, septa and cardiac cushions, and connects the heart with the adjacent vascular endothelial network (Markwald et al. 1975; Markwald et al. 1977; Meyer \& Birchmeier 1995; Kisanuki et al. 2001; Stankunas et al. 2008; Harris \& Black 2010; Red-Horse et al. 2010; X. Tian et al. 2017). Despite these important and unique roles, the developmental cues that distinguish the endocardium from the vascular endothelium remain to be determined.

Genetically, the earliest specific marker of endocardium is Nfatc1 (de la Pompa et al. 1998; Wong et al. 2012). The endocardial-specific expression of this marker, combined with the functionally distinct roles of the endocardium, demonstrate that the endocardium is a unique subset of endothelium with its own transcriptional signature. However, when this distinction is first established at a molecular level remains unclear. In mice, Nfatc1 expression is already detectable in the medial aspect of the cardiac crescent at E7.5 (de la Pompa et al. 1998), whereas in zebrafish, nfatc1 is reported to initiate at 22 hpf, corresponding with the formation of the linear heart tube (Wong et al. 2012). Prior to heart tube formation in zebrafish, endocardial progenitors bud from bilateral, $\mathrm{kdrl}^{+} / \mathrm{fli} \mathrm{a}^{+}$populations at the 12-somite stage (12ss; $15 \mathrm{hpf}$ ) and migrate to the midline to form the endocardial core of the cardiac disc (Bussmann et al. 2007). This observation suggests that the endocardial and vascular endothelial progenitors are functionally distinct as early as the 12ss, when this subset of cells begin migrating to the midline. However, molecular evidence that corroborates this functional distinction has not been reported.

One hypothesis to explain this lack of evidence is that the endocardium develops from a general endothelial progenitor population sequestered within 
56 the cardiac field. In this model, the endothelial progenitors of the heart 57 become encapsulated by myocardium as the cardiac disc forms. The 58 differentiation of the specialised endocardium from this general endothelial 59 population would then occur through myocardial-to-endocardial signalling. As 60 a result, the molecular distinction between the endocardium and the vascular 61 endothelium would occur at, or after, the formation of the cardiac disc, in 62 agreement with the reported expression of nfatc1 (Wong et al. 2012). 63 However, this hypothesis doesn't account for the migration of endocardial, but not endothelial, progenitors to the midline at the 12ss.

The early expression of Nfatc1 in the mouse supports an alternative hypothesis where the endocardium and the vascular endothelium differentiate as unique lineages from an early developmental stage. In agreement with this model, the zebrafish cloche mutant lacks all endocardium, yet retains some endothelium, suggesting that the endocardium and vascular endothelium have distinct developmental origins (Stainier et al. 1995). The cloche mutation was recently mapped to the npas4l gene (Reischauer et al. 2016). Analysis of npas4l expression revealed that expression initiates shortly after gastrulation, at $6 \mathrm{hpf}$, peaks at the tailbud stage, $10 \mathrm{hpf}$, and subsequently reduces to undetectable levels by $24 \mathrm{hpf}$ (Reischauer et al. 2016). Together, this evidence supports the hypothesis that endocardial identity is established early in development.

Here, we report the identification and characterisation of a previously reported marker of the zebrafish lymphatic system as a novel marker of early

79 endocardial progenitors (Bussmann et al. 2010; Okuda et al. 2012). 80 Characterisation of this line, and nfatc1 expression, identified distinct 81 endocardial expression domains as early as the $8 \mathrm{ss}$ (13 hpf). These results 82 confirm the hypothesis that the endocardium and vascular endothelium 83 differentiate as unique lineages from an early developmental stage. 84 Furthermore, using a number of loss-of-function models, we identify both etv2 85 and BMP signalling as important regulators of endocardial differentiation. 86 Further analysis of these factors suggests that BMP signalling acts upstream 
bioRxiv preprint doi: https://doi.org/10.1101/654525; this version posted May 30, 2019. The copyright holder for this preprint (which was

not certified by peer review) is the author/funder, who has granted bioRxiv a license to display the preprint in perpetuity. It is made available under aCC-BY 4.0 International license.

87 of the transcription factor etv2 to direct differentiation of endocardial 88 progenitors. 


\section{Results:}

\section{The Gt(SAGFF27C); $T g(4 x U A S: E G F P)$ line is a marker of early endocardial development}

We obtained the Gt(SAGFF27C); $T g(4 x U A S: E G F P)$ line, originating from a large gene-trap screen (Asakawa et al. 2008), for use as a transgenic reporter of the endothelium. Analysis of 48 hours post fertilisation (hpf) embryos showed fluorescence throughout the endothelium, with stronger fluorescence intensity in the endocardium compared to the adjacent vasculature (figure 1 supplement 1). Examining earlier stages revealed fluorescence in the endocardium at $24 \mathrm{hpf}$ with no obvious signal in the adjacent vascular endothelial network (figure 1 supplement 1), suggesting that, at least at early stages, GFP expression in this line is restricted to the endocardium, providing a novel tool to investigate early endocardial development.

To more carefully characterise the expression of the Gt(SAGFF27C); $\operatorname{Tg}(4 x U A S: E G F P)$ line (henceforth referred to as Gt(endocard:egfp)), an immunofluorescence time course was performed on Gt(endocard:egfp) embryos crossed to the vascular transgenic line, Tg(kdrl:Hsa.HRAS-mCherry) (figure 1). The pan-endothelial Tg(fli1a:EGFP) line was also examined as a basis of comparison (figure 1). Expression of Gt(endocard:egfp) colocalises with Tg(kdrl:Hsa.HRAS-mCherry), confirming that expression for Gt(endocard:egfp) is endothelial (figure 1A). Expression of both Gt(endocard:egfp) and Tg(fli1a:EGFP) lines was observed in bilateral populations of the anterior lateral plate mesoderm as early as the 10 ss (figure 1). The Gt(endocard:egfp) line showed restricted fluorescence in these bilateral populations (figure 1A), whereas the $T g(f l i 1 a: E G F P)$ line had a broader expression domain, extending in the anterior of the embryo, to include presumptive vascular endothelium (figure 1B).

Consistent with previous reports (Bussmann et al. 2007), the bilateral populations of endothelium were found to have migrated to the midline by the 15ss, (figure 1) and by 20-somites have fused to form the endocardial core of the cardiac disc (figure 1 ). In both crosses, a number of $\mathrm{GFP}^{+} / \mathrm{mCherry}^{-}$cells are observed around the cardiac disc. Live timelapse imaging of the 
121 Gt(endocard:egfp) and Tg(fli1a:EGFP) lines indicate that these cells have the

122 morphological and migratory characteristics of myeloid progenitors (figure 1

123 supplement $2-4)$.

124 At $24 \mathrm{hpf}$, endocardial expression is observed in the linear heart tube of 125 Gt(endocard:egfp) embryos (figure 1A). Tg(fli1a:EGFP) and $126 \mathrm{Tg}$ (kdrl:Hsa.HRAS-mCherry) are expressed in the endocardium but, in 127 contrast, also show continuous expression with the adjacent vasculature 128 (figure 1B). By $48 \mathrm{hpf}, \mathrm{Gt}$ (endocard:egfp) expression has expanded and is 129 also observed in the developing vascular endothelium, indicating that this 130 restriction from the vascular endothelium is transient. Additionally, at 24 and $13148 \mathrm{hpf}$ a number of $\mathrm{GFP}^{+} / \mathrm{mCherry}^{-}$cells were observed surrounding the 132 endocardial lining of the heart in Gt(endocard:egfp); $133 \mathrm{Tg}$ (kdrl:Hsa.HRAS-mCherry) but not Tg(fli1a:EGFP); $134 \mathrm{Tg}$ (kdrl:Hsa.HRAS-mCherry) embryos (figure 1A). These $\mathrm{GFP}^{+}$cells 135 expressed noticeably weaker GFP levels than those observed in the 136 endocardial layer and were confirmed to co-localise with the myocardial 137 marker $\operatorname{Tg}($ myl7:mCherry-CaaX) (figure 1 supplement 5), indicating 138 Gt(endocard:egfp) expression in the myocardium at later stages.

139 To investigate the earliest expression of the Gt(endocard:egfp) line, in situ 140 hybridisation was performed over a developmental time-course from the 5ss 141 (11.5 hpf) through to $48 \mathrm{hpf}$ examining gfp transcription (figure 2). gfp 142 expression was first detected as early as the 8ss (13 hpf), this expression was 143 observed in faint semilunar domains in lateral regions at the anterior of the 144 embryo (figure 2) and were lost in a npas4l/cloche mutant (figure 2 145 supplement 1). These bilateral expression domains were observed more 146 posteriorly at the 10ss. By the $15 \mathrm{ss}$ (16.5 hpf), this expression is found at the 147 midline (figure 2) in the region of the endocardial core of the cardiac disc 148 (figure 2), consistent with the observations made by examining fluorescence 149 (figure 1). As gfp expression in this line precedes the reported expression of 150 nfatc1 in zebrafish (Wong et al. 2012), nfatc1 expression was also analysed 151 over the same time-course. Contrary to published reports, nfatc1 expression 152 was also found to initiate as early as the 8ss and was observed in strikingly 
153 similar domains to that of gfp expression in Gt(endocard:egfp) embryos (figure

154 2). Together, these results show that the endocardium begins to express 155 multiple unique markers as early as the 8ss, much earlier than previously 156 appreciated in zebrafish development. This expression is consistent with the 157 observed fluorescence in the Gt(endocard:egfp) line and supports the 158 hypothesis that the endocardial and vascular endothelial lineages diverge 159 during early somitogenesis stages in the vertebrate embryo.

\section{Loss of myocardial and myeloid lineages do not alter early endocardial} progenitor commitment

163 Having established the Gt(endocard:egfp) line as a marker of the 164 endocardium, we sought to confirm its location in relation to other known tissues in the anterior lateral plate mesoderm using two-colour fluorescent in situ hybridisation.

167 Endocardial localisation was examined at the 15ss (16.5 hpf) using probes for 168 gfp expression in Gt(endocard:egfp) embryos along with spi1b or myl7 probes 169 to label myeloid and myocardial populations, respectively (Yelon et al. 1999; 170 Lieschke et al. 2002). Little overlap between gfp and spi1b expression was 171 observed, with myeloid cells positioned anterior and medial to endocardial 172 cells (figure 3 supplement 1 ). A lack of overlapping expression indicates that $173 \mathrm{gfp}$ is not actively transcribed in myeloid cells at the 15ss in Gt(endocard:egfp) 174 embryos, contrasting the GFP fluorescence observed at the protein level 175 (figure 1 supplement 2 - 3). This observation may be explained by perdurance 176 of the GFP. Myocardial expression was located posterior to the endocardium 177 at the 15ss, with no overlap observed between gfp and myl7 expression 178 (figure 3 supplement 1 ).

179 Previous studies have elegantly demonstrated that perturbation of fate180 determining transcription factors can impact the expression domains of 181 myocardial and myeloid populations across the ALPM (Lieschke et al. 2002; 182 Keegan et al. 2005; Schoenebeck et al. 2007; Sumanas et al. 2008; Simões 183 et al. 2011). However, without a unique marker of endocardial identity, this 
184 population is yet to be studied independent of the adjacent myeloid and vascular endothelial lineages.

186

To undertake this, we began by generating a range of loss-of-function models and examined the impact on endocardial development. Surprisingly, neither $n k x 2.5$, hand2 nor tal1 loss-of-function were found to significantly affect the endocardial expression domain at the 14 or $16 \mathrm{ss}$ (16 and $17 \mathrm{hpf}$, respectively; figure 3 supplement 2 - 4). hand2 mutants showed a drastic, but not complete, loss of the myocardial domain as previously reported (Yelon et al. 2000) without altering endocardial or myeloid lineages (figure 3 supplement 3 ). tal1 knockdown caused a significant reduction of the myeloid domain (figure 3 supplement 4), consistent with previous reports (Shivdasani et al. 1995; Gering et al. 1998; Patterson et al. 2005). Interestingly, tal1 knockdown increased the myocardial expression domain and expanded the length of this domain along the anterior-posterior axis without affecting the endocardial domain (figure 3 supplement 4 - 5).

Next, we examined the endocardial regulators npas4l and etv2 in loss-offunction models. As expected, in npas4l mutants endocardial expression was completely lost at both 14 and 16ss when assessed by in situ hybridisation (figure $3 A, E$ ). The myeloid expression domain was also completely lost in mutants (figure 3C), consistent with previous reports (Lieschke et al. 2002), while the myocardial expression domains were expanded (figure 3G, figure 3 supplement 5), as described elsewhere (Schoenebeck et al. 2007).

In etv2 mutants, endocardial expression was decreased as assessed by measuring the surface area of staining at the 14ss (figure 4A - B). Most notably however, the bilateral gfp expression in these mutants was extended along the anterior-posterior axis and localised to the lateral margins of the embryo rather than condensing and migrating to the midline as in wild-type siblings (figure 4A). By the 16ss, the gfp expression domain was markedly reduced (figure 4E - F). In contrast, spi1b expression was lost at the $14 \mathrm{ss}$ and myl7 expression expanded at the 16ss (figure 4C, G). 
214 Together, these results show that the myocardial transcription factors $n k x 2.5$

215 and hand2 are dispensable for early endocardial development. Similarly, tal1

216 was also found to be dispensable, despite its role in maintaining endocardial

217 identity during later development (Van Handel et al. 2012; Schumacher et al.

218 2013). Interestingly, while the myocardial domain was expanded in the

219 absence of endocardial and myeloid domains, no reciprocal relationship was

220 observed with a loss of myocardium in hand2 mutants.

222 BMP signalling is required for endocardial development

223 To identify regulators of endocardial development, a drug screen was

224 performed using a number of inhibitors of major signalling pathways (figure 5

225 supplement 1). Gt(endocard:egfp) embryos were treated with chemical

226 inhibitors, from the $5 \mathrm{ss}(11.5 \mathrm{hpf})$ until fixation at the 14 or $16 \mathrm{ss}$. Fixed

227 embryos were analysed by in situ hybridisation for markers of endocardial,

228 myeloid and myocardial domains as described above.

229 Analysis at the 14ss showed slight decreases in the endocardial expression 230 domain of embryos treated with VEGF, BMP and Wnt inhibitors relative to the 231 DMSO control (with only the VEGF inhibitor treatment reaching statistical 232 significance) (figure 5 supplement 1). By the 16ss, all three inhibitors were 233 found to significantly reduce the endocardial expression domain relative to the 234 DMSO control (figure 5 supplement 1). Inhibition of the Hedgehog pathway 235 slightly increased the endocardial expression domain, however this was not 236 statistically significant.

237 In zebrafish, VEGF signalling has previously been shown to act through Erk 238 during early stages of angiogenesis (Shin, Beane, et al. 2016a; Shin, Male, et 239 al. 2016b). To determine whether VEGF signalling may be acting through Erk 240 in the endocardium, we investigated Erk activity using a phosphorylated Erk 241 (pErk1/2) antibody. Surprisingly, immunofluorescence staining against 242 pErk1/2 in Gt(endocard:egfp) or Tg(fli1a:EGFP) embryos at the 15ss did not 243 show evidence of signalling in the endocardium at this stage (figure 5A - B). 244 We simultaneously examined phosphorylated Smad1/5/8 (pSmad1/5/8), the 
245 downstream effector of BMP signalling (Derynck \& Zhang 2003), and

246 observed compelling pSmad1/5/8 activity in GFP ${ }^{+}$cells in the endocardium at

247 the 15ss using either Gt(endocard:egfp) or Tg(fli1a:EGFP) to co-label

248 endocardium (figure 5C - D). Interestingly, in Tg(fli1a:EGFP) embryos

$249 \mathrm{pSmad} 1 / 5 / 8^{+} / \mathrm{GFP}^{+}$cells did not co-localise with presumptive vascular

250 endothelium (figure 5D), suggesting that BMP signalling is active in the

251 endocardium but not the adjacent vascular endothelium of 15 ss embryos.

252 To examine the role of BMP signalling in endocardial development further, the

253 Gt(endocard:egfp) line was crossed with the heat-shock-inducible transgenic 254 lines, $\operatorname{Tg}$ (hsp70l:bmp2b) or $\operatorname{Tg}$ (hsp70l:noggin3), to either activate or inhibit the 255 BMP pathway (Chocron et al. 2007). Embryos were heat-shocked at the 256 tailbud stage (10 hpf) and fixed at the 14 or $16 \mathrm{ss}$ for analysis of myeloid, 257 endocardial and myocardial lineages (figure 6 and figure 6 supplement 1). 258 Inhibition of the BMP signalling pathway by global induction of noggin3 259 expression resulted in a complete loss of endocardial (figure 6) and myeloid 260 expression domains (figure 6 supplement 1) at the 14ss. The myocardial 261 domain was also severely reduced in this context (figure 6 supplement 1). In 262 the reciprocal experiment, activation of the BMP signalling pathway by global 263 induction of $b m p 2 b$ expression resulted in a significant expansion of the 264 endocardial (figure 6) and myeloid (figure 6 supplement 1) expression 265 domains but did not affect the myocardial domain (figure 6 supplement 1 ). 266 Interestingly, activating BMP signalling not only increased the endocardial 267 expression domain but also resulted in stronger staining compared with 268 siblings lacking the heat-shock transgene (figure 6), indicating both an 269 expansion of the endocardial domain and increased transcription within 270 endocardial cells.

271 Given the requirement of the endocardium for etv2 activity (Figure 4) (Lee et 272 al. 2008; Ferdous et al. 2009; Palencia-Desai et al. 2011), we sought to 273 examine etv2 expression under perturbation of BMP signalling. Activation of 274 BMP signalling by heat-shock at the tailbud stage showed upregulated etv2 275 expression in the anterior lateral plate mesoderm at the 14ss (figure 7). etv2 276 expression in the posterior lateral plate mesoderm was also found to be 
277 upregulated in response to activation of the BMP signalling pathway (figure 7).

278 Reciprocally, inhibition of BMP signalling by heat-shock at the tailbud stage

279 altered the patterning of the expression domain of etv2 in both the anterior 280 and posterior lateral plate mesoderm (figure 7).

281 Together, these results show that BMP signalling plays a crucial role in early 282 endocardial development and is essential for correct etv2 expression, a gene 283 essential for endocardial development. Both BMP inhibition and etv2 loss-of284 function result in a loss of endocardium, suggesting that BMP signalling acts upstream of etv2 to induce endocardial development. 
286

287

288

289

290

\section{Discussion:}

The endocardium is a unique invention of the vertebrate taxa (Pérez-Pomares et al. 2009). Although the endocardium has many molecular similarities to the adjacent vascular endothelium, there are distinct physiological functions of these tissues (Harris \& Black 2010). What distinguishes these lineages on a molecular level remains an important, unanswered question in cardiovascular biology. Using zebrafish as a model for cardiac development, we have identified the Gt(endocard:egfp) gene-trap transgenic line as an endocardial marker and used this to profile the molecular signals required for endocardial differentiation.

Characterisation of the Gt(endocard:egfp) line showed that this marker is uniquely expressed in the progenitors of the endocardium as early as the 8ss (13 hpf) - 9 hours prior to the reported initiation of expression of the endocardial specific marker, nfatc1 (Wong et al. 2012). By re-examining nfatc1 expression in zebrafish embryos it was found that, contrary to previous reports, nfatc1 expression initiates at the same stage as the Gt(endocard:egfp) marker. Although the faint expression of nfatc1 prevents co-localisation by fluorescent in situ hybridisation, these markers are expressed in a strikingly similar spatiotemporal pattern. This pattern is consistent with npas4l expression, the earliest known regulator of endocardial fate, during early somitogenesis stages (Reischauer et al. 2016) and the observed expression domain is lost in the npas $4 l^{\text {uq12ks }}$ mutant. These results demonstrate that the 8ss marks the emergence of definitive markers of the endocardium in the anterior lateral plate mesoderm.

This 8ss is intriguing as tal1 knockdown embryos also develop gene expression defects at this time (Patterson et al. 2005). In tal1 morphants, the mesodermal markers hhex and draculin are substantially reduced from the 7ss, despite initiating expression normally at earlier stages (Patterson et al. 2005). Although our results did not find any association between tal1 loss-offunction and early endocardial development, tal1 has been shown to be required in endocardial development at later stages in zebrafish and mice (Bussmann et al. 2007; Van Handel et al. 2012; Schumacher et al. 2013). 
318 Furthermore, both hhex and draculin are associated with cardiovascular

319 development (Herbomel et al. 1999; Liao et al. 2000; Mosimann et al. 2015;

320 Gauvrit et al. 2018). These results point to the $7 / 8$ ss as a critical phase in the

321 establishment of cardiovascular lineages in zebrafish development.

322 Using gfp expression in the Gt(endocard:egfp) line as a proxy for endocardial 323 gene expression, the relationship between the endocardial, myeloid and 324 myocardial lineages was probed in a number of loss-of-function models. In 325 these models, endocardial and myeloid lineages were regulated in kind in 326 most models examined, suggesting a close association between the 327 development of the endocardial and myeloid lineages in the zebrafish. When 328 endocardial and/or myeloid lineages were lost, there was a resulting increase 329 in the myocardial expression domain, as has previously been reported for 330 cloche mutants (Schoenebeck et al. 2007) and etv2 morphants (Palencia331 Desai et al. 2011). However, no evidence for a reciprocal increase in the 332 endocardial or myeloid lineages was seen when the myocardial domain was 333 ablated in hand2 mutants.

334 While FGF signalling has previously been shown to have reciprocal effects on 335 haemangioblast and myocardial lineages, these effects were most 336 pronounced at the blastula stage (Simões et al. 2011). The results from our 337 experiments, combined with the previous work described above, suggest that 338 there is an early step directing commitment of cardiovascular progenitor cells 339 to the haemangioblast or myocardial lineages. After becoming committed to 340 the haemangioblast programme, additional signals are required to maintain 341 the differentiation of these lineages and repress myocardial development. In 342 the absence of these additional signals, mesodermal cells fail to continue 343 developing along the haemangioblast lineage and default to the myocardial 344 program. In support of this theory, scRNA-seq analysis of mesodermal cells in 345 Tal1 mutant mouse embryos has shown that in the absence of Tal1, 346 haemogenic and endocardial progenitors revert to a myocardial lineage (Van 347 Handel et al. 2012; Scialdone et al. 2016). Moreover, this was not found to be 348 through direct regulation of the myocardial program by Tal1 (Scialdone et al. 349 2016), suggesting that establishment of endocardial identity restrains the 
350 myocardial program and simply halting endocardial differentiation is sufficient

351 to initiate myocardial development.

352 Additional analysis of the endocardial, myeloid and myocardial lineages using 353 inhibitors and genetic tools, as well as the analysis of downstream effectors of 354 signalling pathways, identified the BMP signalling pathway as playing a vital 355 role in the development of the endocardial lineage. Interestingly, inhibition of 356 the BMP signalling pathway in zebrafish by drug treatment from the 5 ss had 357 mild effects on the endocardial and myeloid lineage compared to noggin3 358 over-expression from the tailbud stage. Similarly, while no significant affect 359 was observed on the myocardium by drug treatment from the 5ss, noggin 3 360 over-expression at the tailbud stage resulted in a drastic loss of myocardium. 361 This suggests the early somitogenesis stages mark a crucial window when 362 cardiovascular progenitors transition from an uncommitted to committed state. 363 Furthermore, our results show that BMP signalling is both necessary and 364 sufficient for the development of the endocardial and myeloid lineages, and necessary for the development of the myocardial lineage.

366 The requirement for BMP signalling in endocardial development appears to be 367 conserved in mice, as deletion of the BMP receptor, Alk2, in endothelium but 368 not myocardium results in endocardial defects (Wang et al. 2005). Similarly, 369 activation of Noggin in the endocardium using a constitutively actively 370 Nfatc1 ${ }^{\text {Cre }}$ line results in endocardial-specific defects (Snider et al. 2014). 371 Interestingly, BMP signalling is also sufficient for the maintenance of 372 endocardial identity: zebrafish embryos that are myocardial-deficient fail to 373 express nfatc1, demonstrating myocardium is necessary for the maintenance 374 of endocardial identity. In this context, activation of BMP signalling at 16ss 375 was capable of rescuing nfatc1 expression at 24 and 46 hpf (Palencia-Desai 376 et al. 2015), suggesting that the endocardium is dependent upon BMP 377 signalling, potentially derived from the myocardium at these later stages and 378 from an unknown source at earlier stages. These results support a conserved 379 and ongoing role for BMP signalling in endocardial development.

380 Here, we report that the genetic activation or inhibition of the BMP signalling 381 pathway modulates etv2 expression. As etv2 loss-of-function was also found 
382 to result in a loss of endocardial progenitors, these results have led to a 383 proposed model whereby BMP signalling controls etv2 expression, which in 384 turn directs commitment to the endocardial and myeloid lineages. A number of 385 regulators of etv2 expression have been identified in zebrafish (Veldman \& Lin 386 2012; Schupp et al. 2014), as well as in mice (Rasmussen et al. 2012; 387 Koyano-Nakagawa \& Garry 2017). In support of our hypothesis, BMP 388 signalling has also been shown to regulate etv2 expression through controlling neuromuscular progenitor fate in the zebrafish trunk (Row et al. 2018). Furthermore, two conserved regions in the upstream promoter of 391 murine Etv2 have been identified as containing Smad-binding motifs (Shi et 392 al. 2015). These results suggest that BMP signalling may control etv2 393 expression by direct interaction of Smads with the etv2 promoter. However, it 394 was recently reported that etv2 is directly regulated by npas4l binding to the 395 etv2 locus (Marass et al. 2019). As the timing of npas4l expression and BMP 396 activity are coincident in the embryo, it remains to be determined whether 397 npas4l and Smads function co-operatively, or in parallel, to control the 398 expression of etv2.

399 Together, these results represent the identification of a novel tool for 400 investigating early endocardial development and report defined endocardial 401 expression domains prior to linear heart tube formation in zebrafish. 402 Furthermore, through analysing the endocardial expression domain we have 403 identified BMP signalling as a critical regulator of early endocardial 404 development. Based on these results, we propose a model for endocardial 405 development whereby BMP signalling acts upstream of the transcription factor 406 etv2 to regulate the emergence of endocardial progenitors from the anterior 407 lateral plate mesoderm in zebrafish. 
Materials and Methods:

\section{Zebrafish lines}

410 All zebrafish strains were maintained and animal work performed in 411 accordance with the guidelines of the animal ethics committee at The 412 University of Queensland, Australia. The previously published transgenic lines 413 used in this study are Gt(SAGFF27C); $T g(4 x U A S: G F P)$, referred to as 414 Gt(endocard:egfp) in this text (Bussmann et al. 2010), Tg(kdrl:Hsa.HRAS$415 m$ Cherry) ${ }^{s 916}$ (Hogan et al. 2009), Tg(fli1a:EGFP) ${ }^{y 1}$ (Lawson \& Weinstein 416 2002), Tg(hsp70l:noggin3) $)^{\text {fr14 }}$ and $T g(h s p 70 l: b m p 2 b)^{\text {fr13 }}$ (Chocron et al. 2007).

\section{Morpholino oligonucleotide (MO) reagents}

419 All MOs were ordered from Genetools, LLC. The MO sequences and 420 concentrations used are as follows: $0.8 \mathrm{pmol}$ tal1/scl 421 AATGCTCTTACCATCGTTGATTTCA (Dooley et al. 2005), 1 pmol gata1a 422 CTGCAAGTGTAGTATTGAAGATGTC (Galloway et al. 2005), 1.77 pmol 423 spi1b - GATATACTGATACTCCATTGGTGGT (Rhodes et al. 2005).

\section{CRISPR/Cas9 mutagenesis}

426 To generate the mutant lines used in this study CRISPR/Cas9 mutagenesis 427 was performed as described previously (Gagnon et al. 2014; Capon et al. 428 2016). Embryos and fish were screened for indels by HRMA (Dahlem et al. 429 2012) and carriers sequenced to identify frame-shift mutations that truncate 430 the protein. Specific details of each of the mutants generated for this study are 431 below.

432 The $n k x 2.5$ mutant allele carries a $28 \mathrm{bp}$ deletion in exon one of the $n k x 2.5$ 433 gene and is referred to as the $n k x 2.5^{\text {uq15ks }}$ allele. This mutated allele results in 434 a predicted protein product lacking the DNA binding homeobox domain. An 435 incross of $n k x 2.5^{\text {uq15ks }}$ heterozygous fish produces a mutant phenotype in 
$25 \%$ of progeny at $72 \mathrm{hpf}$ consisting of a cardiac oedema and dilated atrium similar as previous reported (Targoff et al. 2008; Targoff et al. 2013)

The hand 2 mutant allele carries a $4 \mathrm{bp}$ deletion in the first exon of the hand2 gene and is referred to as the hand $2^{\text {uq16ks }}$ allele. The truncated protein product of this mutant allele lacks the DNA binding bHLH domain. Incrossing fish carrying the hand $2^{\text {uq16ks }}$ allele results in a mutant phenotype in $25 \%$ of the progeny in which the affected embryos completely lack a beating heart and have markedly smaller fin buds as previously described (Yelon et al. 2000).

The npas4l mutant allele carries a $7 \mathrm{bp}$ deletion in the second exon of the npas $4 /$ gene and is referred to as the $n p a s 4 l^{\text {uq } 14 k s}$ allele. The predicted protein product of this truncated allele partially retains the DNA-binding bHLH domain, but lacks the two PAS domains as well as the three transcriptional activation domains. An incross of fish carrying the npas $4 l^{\text {uq } 14 k s}$ allele produces a mutant phenotype in $25 \%$ of the progeny, consisting of a bell-shaped heart and lacking the endocardial layer at $48 \mathrm{hpf}$ as previously described for the cloche mutant (Stainier et al. 1995).

The etv2 mutant allele has a $23 \mathrm{bp}$ deletion in the exon five of the etv2 gene and is referred to as the etv2 ${ }^{\text {uq13ks }}$ allele. This truncated allele completely lacks the DNA-binding ETS domain. An incross of fish heterozygous for the etv $2^{\text {uq13ks }}$ allele produces a mutant phenotype in $25 \%$ of progeny. This phenotype is characterised by a lack of blood circulation, as previously reported (Pham et al. 2007) as well as a collapsed heart at 48 hpf. Interestingly, the cardiac defect at $48 \mathrm{hpf}$ was variable with the most severely affected mutant embryos lacking the majority of the endocardium.

\section{In situ hybridisation}

In situ hybridisation was performed as previously described (C. Thisse \& B. Thisse 2008). All RNA probes were transcribed from plasmids. Plasmids containing the spi $1 b$ and gfp coding sequences were a kind gift from the laboratory of Ben Hogan. To generate the etv2 plasmid used in this study, 
466

467

468

469

470

pCS2+ vector was prepared by digesting empty vector with BamHI and $\mathrm{Xbal}$. The etv2 cDNA sequence was amplified by PCR using the primer sequences below, the PCR product was purified and inserted into the digested pCS2+ vector using circular polymerase extension cloning (Quan \& J. Tian 2011).

etv2_cDNA_F:

AAGCTACTTGTTCTTTTTGCAGGATCTGTCAAAACCCCTGATATAGTG

etv2_cDNA_R:

\section{TGGATCTACGTAATACGACTCACTATAGTTCTAGCAATCTGCTGCAAAGT}

CC

\section{Fluorescent in situ hybridisation}

Fluorescent in situ hybridisation was performed as previously described (Clay \& Ramakrishnan 2005; Brend \& Holley 2009; Baek et al. 2019) with minor alterations. In brief, embryos were dechorionated and fixed at the desired stage. RNA probes were synthesised with DIG or FLU RNA labelling mix (Roche). Fixed embryos were permeabilised with proteinase K (Invitrogen) and hybridised with $1 \mathrm{ng} / \mu \mathrm{L}$ RNA probe in hybridisation buffer overnight at $70^{\circ} \mathrm{C}$. After hybridisation, embryos were washed, blocked with western blocking reagent (Roche) and incubated with anti-dig or anti-flu, POD antibodies (Roche) in western blocking reagent overnight at $4^{\circ} \mathrm{C}$. After further washes, staining was performed using the tyramide signalling amplification kit (Perkin Elmer) for 2 hours at $37^{\circ} \mathrm{C}$. Following staining embryos were fixed at $4^{\circ} \mathrm{C}$ overnight and mounted for imaging.

\section{Heat-shock treatment}

Heat-shock was performed at $39^{\circ} \mathrm{C}$ for 30 minutes in pre-warmed medium. Following heat-shock, embryos were returned to a $28.5^{\circ} \mathrm{C}$ incubator until fixation. 


\section{Antibody staining}

495 Phospho-protein staining was performed as previously described (Okuda et 496 al. 2018) using the following antibodies: GFP (abcam cat. no. ab13970), 497 pErk1/2 (cell signaling technologies cat. no. 4370), pSmad1/5/8 (cell signaling 498 technologies cat. no. 13820).

\section{Drug treatments}

501 Chemical inhibitors of the following signalling pathways were ordered from 502 Merck: cyclopamine - hedgehog inhibitor (cat. no. C4116), DAPT - Notch 503 inhibitor (cat. no. D5942), DMH1 - BMP inhibitor (cat. no. D8946), IWR1 - Wnt 504 inhibitor (cat. no. 10161), SU5416 - VEGF inhibitor (cat. no. S8442). These 505 inhibitors were dissolved in DMSO, aliquoted, and stored at $-20^{\circ} \mathrm{C}$. For 506 treatments, inhibitors were dissolved in E3 medium to the indicated 507 concentration below, added to embryos at a ratio of 15 embryos $/ \mathrm{mL}$ and 508 incubated in the dark at $28.5^{\circ} \mathrm{C}$ until fixation.

\begin{tabular}{ccc}
\hline Drug name & Signalling pathway inhibited & Working concentration \\
\hline Cyclopamine & Hedgehog & $50 \mu \mathrm{M}$ \\
DAPT & Notch & $100 \mu \mathrm{M}$ \\
DMH1 & BMP & $1 \mu \mathrm{M}$ \\
IWR1 & Wnt & $10 \mu \mathrm{M}$ \\
SU5416 & VEGF & $1 \mu \mathrm{M}$ \\
\hline
\end{tabular}

509

511 Embryos from in situ hybridisation were dehydrated, cleared in murray's

512 solution (a 2:1 ratio of benzyl benzoate:benzyl alcohol) and imaged using an

513 Olympus BX51 Microscope with Olympus DP70 CCD camera.

514 For wide-field bright-field and fluorescence imaging, embyros were mouted in $5153 \%$ methyl cellulose (Sigma, cat. no. M0387) and imaged using a Leica M165 516 FC stereo microscope with a DFC425 C camera. 
517 All confocal imaging was performed on a Zeiss LSM 710 FCS confocal

518 microscope. Live embryos were mounted for confocal imaging using $0.5-1 \%$

519 low-melting agarose (Sigma, cat. no. A9414) in glass-bottom petri dishes.

520 Fixed embryos for confocal imaging were de-yolked using lash tools and

521 mounted in vectashield with DAPI (Vector laboratories, cat. no. $\mathrm{H}-1200$ ) on

522 glass-slides with coverslips.

523 All images were processed using FIJI (Schindelin et al. 2012) and/or Imaris 524 software.

\section{Statistical testing}

527 A student's t-test with Welch's correction was performed using Prism 528 software. For data-sets containing 3 or more conditions, one-way ANOVA

529 testing with Tukey's post hoc test for multiple comparisons was performed 530 using Prism software. 


\section{Acknowledgements:}

532 We acknowledge members of the Smith laboratory for useful discussions and

533 Ben Hogan and lab members for discussions and sharing of reagents. We

534 thank the UQBR aquatics team for animal husbandry. Confocal microscopy

535 was performed at the Australian Cancer Research Foundation's Cancer

536 Ultrastructure and Function Facility at the IMB.

538 Funding:

539 SJC was supported by an Australian Government Australian Postgraduate

540 Award (APA) and KAS was part-funded by an Australian Research Council 541 (ARC) Future Fellowship (FT110100496). The research was funded by an 542 ARC Discovery Project grant (DP170101217).

\section{Competing interests:}

545 The authors declare that no competing interests exist. 


\section{References:}

547 Asakawa, K. et al., 2008. Genetic dissection of neural circuits by Tol2 transposon-mediated Gal4 gene and enhancer trapping in zebrafish. America, 105(4), pp.1255-1260.

Baek, S. et al., 2019. The Alternative Splicing Regulator Nova2 Constrains Vascular Erk Signaling to Limit Specification of the Lymphatic Lineage. Developmental Cell, 49(2), pp.279-292.e5.

Brend, T. \& Holley, S.A., 2009. Zebrafish whole mount high-resolution double fluorescent in situ hybridization. Journal of visualized experiments : JoVE, (25).

Bussmann, J. et al., 2010. Arteries provide essential guidance cues for lymphatic endothelial cells in the zebrafish trunk. Development, 137(16), pp.2653-2657.

Bussmann, J., Bakkers, J. \& Schulte-Merker, S., 2007. Early endocardial morphogenesis requires Scl/Tal1. PLoS genetics, 3(8), p.e140.

Capon, S.J. et al., 2016. Utilising polymorphisms to achieve allele-specific genome editing in zebrafish. Biology Open, p.bio.020974.

564 Chocron, S. et al., 2007. Zebrafish Bmp4 regulates left-right asymmetry at two distinct developmental time points. Developmental Biology, 305(2), pp.577-588.

567 Clay, H. \& Ramakrishnan, L., 2005. Multiplex fluorescent in situ hybridization 568 in zebrafish embryos using tyramide signal amplification. Zebrafish, 2(2), pp.105-111.

570 Dahlem, T.J. et al., 2012. Simple methods for generating and detecting locus571 specific mutations induced with TALENs in the zebrafish genome. PLoS genetics, 8(8), p.e1002861.

573 de la Pompa, J.L. et al., 1998. Role of the NF-ATc transcription factor in 
574

575

576

577

578

579

580

581

582

583

584

585

586

587

588

589

590

591

592

593

594

595

596

597

598

599

600

morphogenesis of cardiac valves and septum. Nature, 392(6672), pp.182186.

Derynck, R. \& Zhang, Y.E., 2003. Smad-dependent and Smad-independent pathways in TGF-beta family signalling. Nature, 425(6958), pp.577-584.

Dooley, K.A., Davidson, A.J. \& Zon, L.I., 2005. Zebrafish scl functions independently in hematopoietic and endothelial development. Developmental Biology, 277(2), pp.522-536.

Ferdous, A. et al., 2009. Nkx2-5 transactivates the Ets-related protein 71 gene and specifies an endothelial/endocardial fate in the developing embryo. Proceedings of the National Academy of Sciences of the United States of America, 106(3), pp.814-819.

Gagnon, J.A. et al., 2014. Efficient mutagenesis by Cas9 protein-mediated oligonucleotide insertion and large-scale assessment of single-guide RNAs. PLoS ONE, 9(5), p.e98186.

Galloway, J.L. et al., 2005. Loss of gata1 but not gata2 converts erythropoiesis to myelopoiesis in zebrafish embryos. Developmental Cell, 8(1), pp.109-116.

Gauvrit, S. et al., 2018. HHEX is a transcriptional regulator of the VEGFC/FLT4/PROX1 signaling axis during vascular development. Nature communications, 9(1), p.2704.

Gering, M. et al., 1998. The SCL gene specifies haemangioblast development from early mesoderm. The EMBO journal, 17(14), pp.4029-4045.

Harris, I.S. \& Black, B.L., 2010. Development of the Endocardium. Pediatric Cardiology, 31(3), pp.391-399.

Herbomel, P., Thisse, B. \& Thisse, C., 1999. Ontogeny and behaviour of early macrophages in the zebrafish embryo. Development, 126(17), pp.37353745.

601 Hogan, B.M. et al., 2009. Ccbe1 is required for embryonic lymphangiogenesis 
and venous sprouting. Nature genetics, 41(4), pp.396-398.

603 Keegan, B.R. et al., 2005. Retinoic acid signaling restricts the cardiac progenitor pool. Science (New York, N.Y.), 307(5707), pp.247-249.

Kisanuki, Y.Y. et al., 2001. Tie2-Cre transgenic mice: a new model for 606 endothelial cell-lineage analysis in vivo. Developmental Biology, 230(2), pp.230-242.

Koyano-Nakagawa, N. \& Garry, D.J., 2017. Etv2 as an essential regulator of mesodermal lineage development. Cardiovascular Research, 113(11), pp.1294-1306.

Lawson, N.D. \& Weinstein, B.M., 2002. In vivo imaging of embryonic vascular development using transgenic zebrafish. Developmental Biology, 248(2), pp.307-318.

Lee, D. et al., 2008. ER71 acts downstream of BMP, Notch, and Wnt signaling

Liao, W. et al., 2000. Hhex and scl function in parallel to regulate early endothelial and blood differentiation in zebrafish. Development, 127(20), pp.4303-4313.

Lieschke, G.J. et al., 2002. Zebrafish SPI-1 (PU.1) marks a site of myeloid development independent of primitive erythropoiesis: implications for axial patterning. Developmental Biology, 246(2), pp.274-295.

Marass, M. et al., 2019. Genome-wide strategies reveal target genes of Npas4l associated with vascular development in zebrafish. Development.

Markwald, R.R., Fitzharris, T.P. \& Manasek, F.J., 1977. Structural development of endocardial cushions. The American journal of anatomy,

628 Markwald, R.R., Fitzharris, T.P. \& Smith, W.N., 1975. Sturctural analysis of 
180.

631 Meyer, D. \& Birchmeier, C., 1995. Multiple essential functions of neuregulin in development. Nature, 378(6555), pp.386-390.

Mosimann, C. et al., 2015. Chamber identity programs drive early functional partitioning of the heart. Nature communications, 6, p.8146.

Okuda, K.S. et al., 2012. Iyve1 expression reveals novel lymphatic vessels and new mechanisms for lymphatic vessel development in zebrafish. Development, 139(13), pp.2381-2391.

Okuda, K.S., Baek, S. \& Hogan, B.M., 2018. Visualization and Tools for Analysis of Zebrafish Lymphatic Development. Methods in molecular biology (Clifton, N.J.), 1846, pp.55-70.

Palencia-Desai, S. et al., 2015. Myocardium and BMP signaling are required for endocardial differentiation. Development.

Palencia-Desai, S. et al., 2011. Vascular endothelial and endocardial progenitors differentiate as cardiomyocytes in the absence of Etsrp/Etv2 function. Development, 138(21), pp.4721-4732.

Patterson, L.J., Gering, M. \& Patient, R., 2005. Scl is required for dorsal aorta as well as blood formation in zebrafish embryos. Blood, 105(9), pp.35023511.

Pérez-Pomares, J.M., González-Rosa, J.M. \& Muñoz-Chápuli, R., 2009. Building the vertebrate heart - an evolutionary approach to cardiac development. The International journal of developmental biology, 53(810), pp.1427-1443.

Pham, V.N. et al., 2007. Combinatorial function of ETS transcription factors in the developing vasculature. Developmental Biology, 303(2), pp.772-783.

655 Quan, J. \& Tian, J., 2011. Circular polymerase extension cloning for high656 throughput cloning of complex and combinatorial DNA libraries. Nature protocols, 6(2), pp.242-251. 
658 Rasmussen, T.L. et al., 2012. VEGF/Flk1 signaling cascade transactivates Etv2 gene expression. PLoS ONE, 7(11), p.e50103.

660 Red-Horse, K. et al., 2010. Coronary arteries form by developmental reprogramming of venous cells. Nature, 464(7288), pp.549-553.

Reischauer, S. et al., 2016. Cloche is a bHLH-PAS transcription factor that 663 drives haemato-vascular specification. Nature, 535(7611), pp.294-298.

664 Rhodes, J. et al., 2005. Interplay of pu.1 and gata1 determines myelo665 erythroid progenitor cell fate in zebrafish. Developmental Cell, 8(1), 666 pp.97-108.

Row, R.H. et al., 2018. BMP and FGF signaling interact to pattern mesoderm by controlling basic helix-loop-helix transcription factor activity. eLife, 7.

Schindelin, J. et al., 2012. Fiji: an open-source platform for biological-image analysis. Nature methods, 9(7), pp.676-682.

Schoenebeck, J.J., Keegan, B.R. \& Yelon, D., 2007. Vessel and blood 672 specification override cardiac potential in anterior mesoderm. Developmental Cell, 13(2), pp.254-267.

Schumacher, J.A. et al., 2013. tal1 Regulates the formation of intercellular 675 junctions and the maintenance of identity in the endocardium. 676 Developmental Biology, 383(2), pp.214-226.

Schupp, M.-O. et al., 2014. Transcriptional inhibition of etv2 expression is essential for embryonic cardiac development. Developmental Biology,

Scialdone, A. et al., 2016. Resolving early mesoderm diversification through single-cell expression profiling. Nature, 535(7611), pp.289-293.

682 Shi, X. et al., 2015. The transcription factor, Mesp1, interacts with cAMP 683 responsive element binding protein 1 (Creb1) and coactivates Ets variant 6842 (Etv2) gene expression. The Journal of biological chemistry. 
685

686

687

688

689

690

691

692

693

694

695

696

697

698

699

700

701

702

703

704

705

706

707

708

709

710

Shin, M., Beane, T.J., et al., 2016a. Vegfa signals through ERK to promote angiogenesis, but not artery differentiation. Development, 143(20), pp.3796-3805.

Shin, M., Male, I., et al., 2016b. Vegfc acts through ERK to induce sprouting and differentiation of trunk lymphatic progenitors. Development, 143(20), pp.3785-3795.

Shivdasani, R.A., Mayer, E.L. \& Orkin, S.H., 1995. Absence of blood formation in mice lacking the T-cell leukaemia oncoprotein tal-1/SCL. Nature, 373(6513), pp.432-434.

Simões, F.C., Peterkin, T. \& Patient, R., 2011. Fgf differentially controls crossantagonism between cardiac and haemangioblast regulators. Development, 138(15), pp.3235-3245.

Snider, P. et al., 2014. Ectopic Noggin in a Population of Nfatc1 Lineage Endocardial Progenitors Induces Embryonic Lethality. Journal of cardiovascular development and disease, 1(3), pp.214-236.

Stainier, D.Y.R. et al., 1995. cloche, an early acting zebrafish gene, is required by both the endothelial and hematopoietic lineages. Development, 121(10), pp.3141-3150.

Stankunas, K. et al., 2008. Endocardial Brg1 represses ADAMTS1 to maintain the microenvironment for myocardial morphogenesis. Developmental Cell, 14(2), pp.298-311.

Sumanas, S. et al., 2008. Interplay among Etsrp/ER71, Scl, and Alk8 signaling controls endothelial and myeloid cell formation. Blood, 111(9), pp.4500-4510.

Targoff, K.L. et al., 2013. Nkx genes are essential for maintenance of ventricular identity. Development, 140(20), pp.4203-4213.

711 Targoff, K.L., Schell, T. \& Yelon, D., 2008. Nkx genes regulate heart tube 712 extension and exert differential effects on ventricular and atrial cell 
number. Developmental Biology, 322(2), pp.314-321.

714 Thisse, C. \& Thisse, B., 2008. High-resolution in situ hybridization to wholemount zebrafish embryos. Nature protocols, 3(1), pp.59-69.

716 Tian, X. et al., 2017. Identification of a hybrid myocardial zone in the 717 mammalian heart after birth. Nature communications, 8(1), p.87.

718 Van Handel, B. et al., 2012. Scl represses cardiomyogenesis in prospective 719 hemogenic endothelium and endocardium. Cell, 150(3), pp.590-605.

720 Veldman, M.B. \& Lin, S., 2012. Etsrp/Etv2 is directly regulated by Foxc1a/b in 721 the zebrafish angioblast. Circulation research, 110(2), pp.220-229.

722 Wang, J. et al., 2005. Atrioventricular cushion transformation is mediated by 723 ALK2 in the developing mouse heart. Developmental Biology, 286(1), 724 pp.299-310.

725 Wong, K.S. et al., 2012. Hedgehog signaling is required for differentiation of 726 endocardial progenitors in zebrafish. Developmental Biology, 361(2), pp.377-391.

728 Yelon, D., Horne, S.A. \& Stainier, D.Y., 1999. Restricted expression of cardiac myosin genes reveals regulated aspects of heart tube assembly in zebrafish. Developmental Biology, 214(1), pp.23-37.

731 Yelon, D. et al., 2000. The bHLH transcription factor hand2 plays parallel roles 732 in zebrafish heart and pectoral fin development. Development, 127(12), 733 pp.2573-2582. 
Figures:

735 Figure 1. Timecourse analysis of Gt(endocard:egfp) shows restricted 736 endocardial expression at somitogenesis stages in the zebrafish 737 embryo. A. Immunofluorescence staining of Gt(endocard:egfp); 738 Tg(kdrl:Hsa.HRAS-mCherry) and B. Tg(fli1a:egfp); Tg(kdrl:Hsa.HRAS739 mCherry) embryos from the 10ss through to $48 \mathrm{hpf}$. Anterior to the top. 740 Anterior views at $48 \mathrm{hpf}$, all other images show dorsal views. White 741 arrowheads label fli $1 \mathrm{a}^{+} / \mathrm{kdrl}^{+} / \mathrm{SAGFF} 27 \mathrm{C}^{+}$endocardial cells. White arrows 742 label $\mathrm{fli} \mathrm{a}^{+} / \mathrm{kdrl}^{+} / \mathrm{SAGFF} 27 \mathrm{C}^{-}$vascular endothelial cells. Black arrowheads 743 label presumptive myocardial cells. Scale bars represent $50 \mu \mathrm{m}$.

Figure 2. Restricted endocardial markers are first expressed at the 8ss in zebrafish embryos. In situ hybridisation for gfp expression in Gt(endocard:egfp) embryos and nfatc1 expression from the $5 \mathrm{ss}$ (11.5 hpf)

748 through to $48 \mathrm{hpf}$, showing expression emerging from 8ss onwards. White 749 arrowheads show the expression domains corresponding to endocardial cells 750 and their progenitors. Anterior to the top for $5 s s-24 \mathrm{hpf}$. Anterior views at 48 $751 \mathrm{hpf}$, all other images show dorsal views. Scale bars represent $100 \mu \mathrm{m}$. The 752 number of embryos matching the image is indicated in the top right of each 753 image. Figure 3. Gt(endocard:egfp) expression is lost in cloche/npas4l mutants.

756 In situ hybridisation for A. gfp and C. spi1b expression at the 14ss and E. gfp 757 and G. myl7 expression at the 16 ss in $n p a s 4 l^{\text {uq14ks }}$ mutants, heterozygotes 758 and wildtype siblings. Quantification of expression domain is shown in $\mathbf{B}, \mathbf{D}, \mathbf{F}$ 759 and H. Dorsal views, with anterior to the top. Scale bars represent $100 \mu \mathrm{m}$. P 760 values are present in graphs. 
762 Figure 4. Gt(endocard:egfp) expression is reduced in etv2 mutants. In

763 situ hybridisation for A. gfp and C. spi1b expression at the 14ss and E. gfp

764 and G. myl7 expression at the $16 \mathrm{ss}$ in etv2 ${ }^{\text {uq13ks }}$ mutants, heterozygotes and

765 wildtype siblings. Quantification of expression domain is shown in B, D, F and

766 H. Dorsal views, with anterior to the top. Scale bars represent $100 \mu \mathrm{m}$. P

767 values are present in graphs.

Figure 5. BMP is actively signalling in developing endocardial cells. Immunofluorescence staining for pErk1/2 in A. Gt(endocard:egfp) and B.

$771 \mathrm{Tg}$ (fli1a:egfp) embryos at the 15ss shows minimal pErk1/2 signal in 772 endocardial cells and high activity in adjacent vasculature. By contrast,

773 pSmad1/5/8 in C. Gt(endocard:egfp) and D. Tg(fli1a:egfp) embryos at 15ss 774 shows high pSmad1/5/8 activity in developing endocardial cell but minimal 775 activity in adjacent vasculature. Dorsal views are shown with anterior to the 776 top in all images. Scale bars represent $50 \mu \mathrm{m}$.

Figure 6. BMP signalling is required for endocardial development. In situ hybridisation for endocardial markers A. gfp (in Gt(endocard:egfp) embryos)

780 and C. nfatc1 in wildtype (transgenic negative siblings) or $T g$ (hsp70l:noggin3)

781 embryos at the 14ss (heat-shocked performed at tailbud stage (10 hpf)) show 782 significantly reduced staining of the endocardial domain upon inhibition of 783 BMP signaling. B and D. Quantification of the area of expression shown in A 784 and C. Reciprocally, wildtype sibling controls or $\operatorname{Tg}($ hsp70l:bmp2b) embryos at 785 14ss (heat-shocked at tailbud) show increased staining area in E. 786 Gt(endocard:egfp) embryos stained for $g f p$ or G. embryos stained for nfatc1. F 787 and $\mathbf{H}$. Quantification of the area of expression shown in $\mathbf{E}$ and $\mathbf{G}$. Dorsal 788 views are shown with anterior to the top in all images. Scale bars represent $789100 \mu \mathrm{m}$. $\mathrm{P}$ values are present in graphs. 
791 Figure 7. etv2 expression is altered by BMP signaling modulation. In situ

792 hybridisation for etv2 expression in wildtype sibling controls,

793 Tg(hsp70l:noggin3) or Tg(hsp70l:bmp2b) embryos. The etv2 expression

794 domain is altered with inhibition of BMP signaling and expanded and

795 upregulated with overactivation of BMP signaling. Dorsal views are shown

796 with anterior to the top in all images. 9/9 Tg(hsp70l:noggin3) embryos

797 matched the expression pattern shown while 11/14 WT embryos matched the

798 WT pattern shown. 13/13 Tg(hsp70l:bmp2b) embryos matched the expression

799 pattern shown while 10/10 WT embryos matched the WT pattern shown Scale

800 bars represent $100 \mu \mathrm{m}$. ALPM - anterior lateral plate mesoderm, PLPM -

801 posterior lateral plate mesoderm. 


\section{Supplemenatary material:}

803 Figure 1 supplement 1. Live lateral view imaging of Gt(endocard:egfp) 804 embryos at 24 and $48 \mathrm{hpf}$, A. with and B. without brightfield. Each panel 805 shows a homozygous and heterozygous embryo imaged side-by-side in a 806 single image. A low-GFP fluorescing embryo, heterozygous for both 807 Gt(SAGFF27C) and $T g(U A S: G F P)$ transgenes, is shown above a high-GFP 808 fluorescing embryo, homozygous for both Gt(SAGFF27C) and $\operatorname{Tg}(U A S: G F P)$ 809 transgenes. Composite images are shown in A while GFP only is shown in B. Scale bars represent $500 \mu \mathrm{m}$.

812 Figure 1 supplement 2. Live, time-lapse imaging of a representative 813 Gt(endocard:egfp) embryo beginning at the 11-somite stage. Confocal z814 stacks were acquired at 5-minute intervals over a 4-hour period. Dorsal views 815 are shown with anterior to the top. The bilateral populations of endocardial 816 progenitors are observed emerging in the lateral margins of the ALPM before 817 rapidly migrating to the midline to form the endocardial core of the cardiac 818 disc. A small number of myeloid cells are observed emerging from the ALPM simultaneously with the endocardial progenitors and migrating away from the midline.

Figure 1 supplement 3 . Live, time-lapse imaging of a representative $\operatorname{Tg}$ (fli1a:egfp) embryo beginning at the 12-somite stage. Confocal z-stacks were acquired at 5 minute intervals over a 4 hour period. Dorsal views are

825 shown with anterior to the top. Bilateral populations of endocardial progenitors 826 are observed migrating to the midline to form the endocardial core of the 827 cardiac disc. Compared with Gt(endocard:egfp) embryos, a large number of 828 myeloid cells are observed emerging from the ALPM and migrating away from 829 the midline. A number of GFP positive cells are also observed in the lateral 830 margins forming the cranial vascular endothelium while endothelial 831 progenitors from the trunk are observed coming into frame as the migrate 832 anteriorly. 
833 Figure 1 supplement 4. Still images from a representative time-lapse

834 imaging on a Tg(fli1a:EGFP) embryo from the 12-somite stage (15 hpf) (A - E)

835 and a representative Gt(endocard:egfp) embryo from the 11-somite stage

836 (14.5 hpf) (F - J). Z-stacks were acquired at 5 minute intervals over a 4 hour

837 period. Dorsal view is shown with the anterior to the top. Orange arrowheads

838 point to emerging endocardial progenitors. Orange arrows point to migratory

839 myeloid and vascular endothelial cells. Orange asterisk shows the

840 endocardial progenitors after merging in the midline.

841

842 Figure 1 supplement 5. Live imaging of Gt(endocard:egfp);

$843 \mathrm{Tg}$ (myl7:mCherry-CaaX) embryos at $48 \mathrm{hpf}$. A Z-projection of the full heart is

844 shown. Selected slices of the ventricle are also shown. All images are ventral 845 views with anterior to the top. Scale bars represent $50 \mu \mathrm{m}$ (in Z-projection) 846 and $25 \mu \mathrm{m}$ (in slices). White arrowheads label endocardium, white arrows 847 label myocardium.

849 Figure 2 supplement 1. In situ hybridisation for endocardial gfp expression in 850 Gt(endocard:egfp); npas4I WT sibling and homozygous mutant embryos at 851 the 8ss (13 hpf). Dorsal views in all images. Scale bars represent $100 \mu \mathrm{m}$. 852 The number of embryos matching the image is indicated in the top right of 853 each image.

Figure 3 supplement 1. Fluorescent in situ hybridisation for gfp expression in marker, spi1b $(\mathrm{n}=3)$, or the myocardial marker, myl7 $(\mathrm{n}=3)$. Embryos were collected at the $15 \mathrm{ss}(16.5 \mathrm{hpf})$. Dorsal views are shown with anterior to the top in all images. Scale bars represent $100 \mu \mathrm{m}$. 
861 Figure 3 supplement 2. In situ hybridisation for endocardial gfp $(A, E)$,

862 myeloid, spi1b (C), and myocardial, myl7 (G), markers in $n k x 2.5^{\text {uq15ks }}$ mutants 863 and siblings. Quantification of the expression is shown in $B, D, F$ and $H$.

864 Dorsal views are shown with anterior to the top in all images. Scale bars 865 represent $100 \mu \mathrm{m}$. P values are indicated in graphs.

Figure 3 supplement 3 . In situ hybridisation for endocardial gfp $(A, E)$, myeloid, spi1b $(\mathrm{C})$, and myocardial, myl7 $(\mathrm{G})$, markers in hand $2^{\text {uq16ks }}$ mutants and siblings. Quantification of the expression is shown in $B, D, F$ and $H$. Dorsal views are shown with anterior to the top in all images. Scale bars represent $100 \mu \mathrm{m}$. $P$ values are indicated in graphs.

Figure 3 supplement 4. In situ hybridisation for endocardial gfp (A,E), 874 myeloid, spi1b (C), and myocardial, myl7 (G), markers in tal1 morphants 875 injected with $0.8 \mathrm{pmol} \mathrm{MO}$ and uninjected control (UIC) embryos. 876 Quantification of the expression is shown in B, D, F and H. Dorsal views are 877 shown with anterior to the top in all images. Scale bars represent $100 \mu \mathrm{m}$. P 878 values are indicated in graphs.

880 Figure 3 supplement 5. The length of the myocardial expression domains, as 881 defined by myl7 expression, were measured along the anterior-posterior axis 882 in $n p a s 4 l^{\text {uq14ks }}(\mathrm{A})$, etv2 $2^{\text {uq13ks }}(\mathrm{B}), n k \times 2.5^{\text {uq15ks }}(\mathrm{C})$, and hand $2^{\text {uq16ks }}$ mutants (D) as well as in tal1 (F) morphants. $P$ values are indicated in graphs.

Figure 5 supplement 1 . The effect of inhibiting a number of signalling pathways on the formation of endocardial, myeloid and myocardial expression domains as assessed by gfp, spi1b and myl7 expression. Drugs were added

888 at the 5ss and embryos incubated until the 14- or 16-ss. Quantification of 889 expression of relevant markers was examined by in situ hybridisation staining 
890 and surface area measurements as previously described. P values are 891 indicated in graphs.

892

893 Figure 6 supplement 1. In situ hybridisation for myeloid, spi1b $(A, G)$, 894 endocardial, gfp (C,I), and myocardial, myl7 $(\mathrm{E}, \mathrm{K})$, markers in embryos from 895 crosses of the Gt(endocard:egfp) line to either the $\operatorname{Tg}$ (hsp70:noggin3) or $896 \operatorname{Tg}(h s p 70: b m p 2 b)$ lines. Quantification of the expression is shown in B, D, F, $897 \mathrm{H}, \mathrm{J}$ and L. Dorsal views are shown with anterior to the top in all images. 898 Scale bars represent $100 \mu \mathrm{m}$. P values are indicated in graphs. 


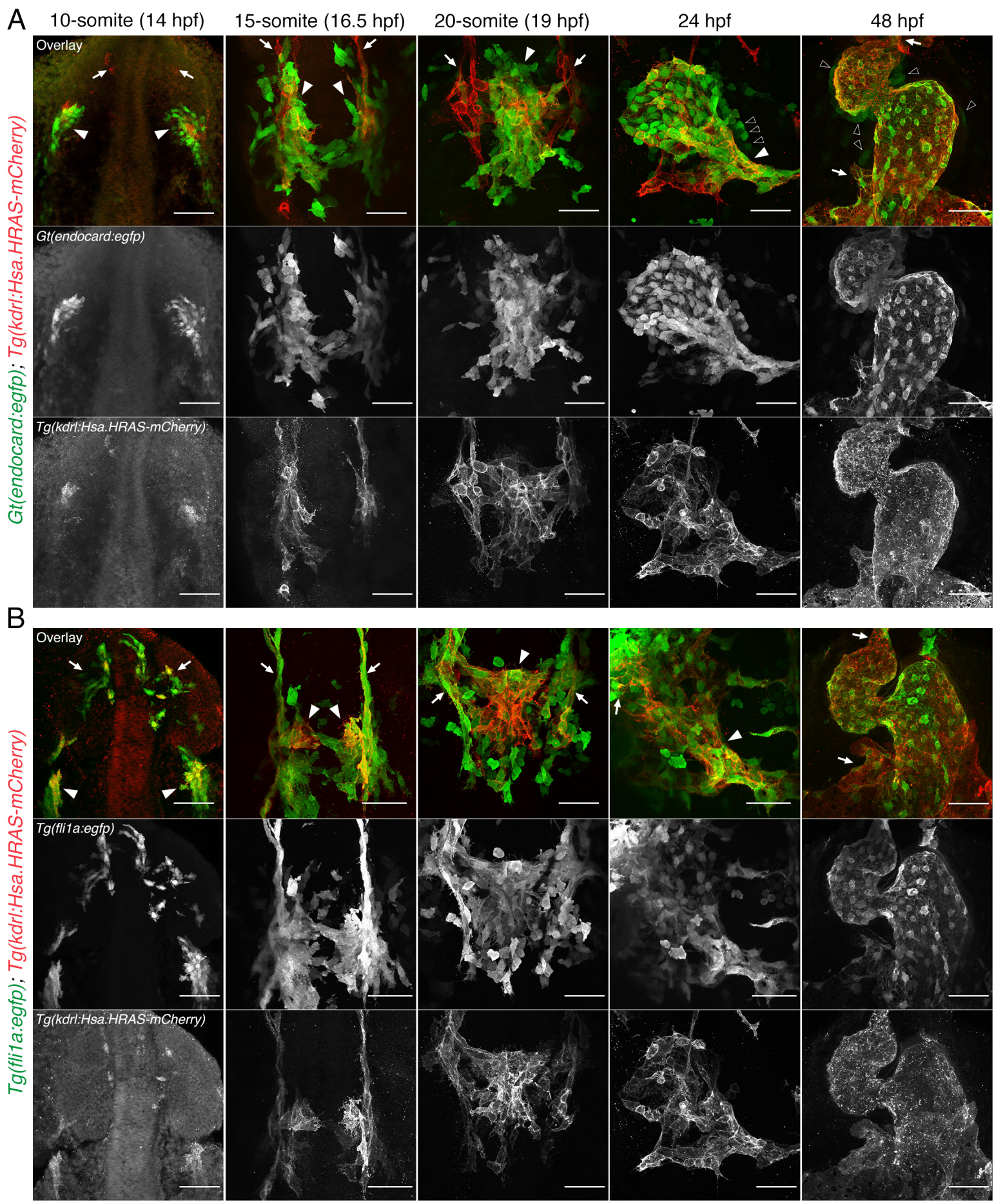

Figure 1. Timecourse analysis of Gt(endocard:egfp) shows restricted endocardial expression at somitogenesis stages in the zebrafish embryo. 
bioRxiv preprint doi: https://doi.org/10.1101/654525; this version posted May 30, 2019. The copyright holder for this preprint (which was not certified by peer review) is the author/funder, who has granted bioRxiv a license to display the preprint in perpetuity. It is made available under aCC-BY 4.0 International license.

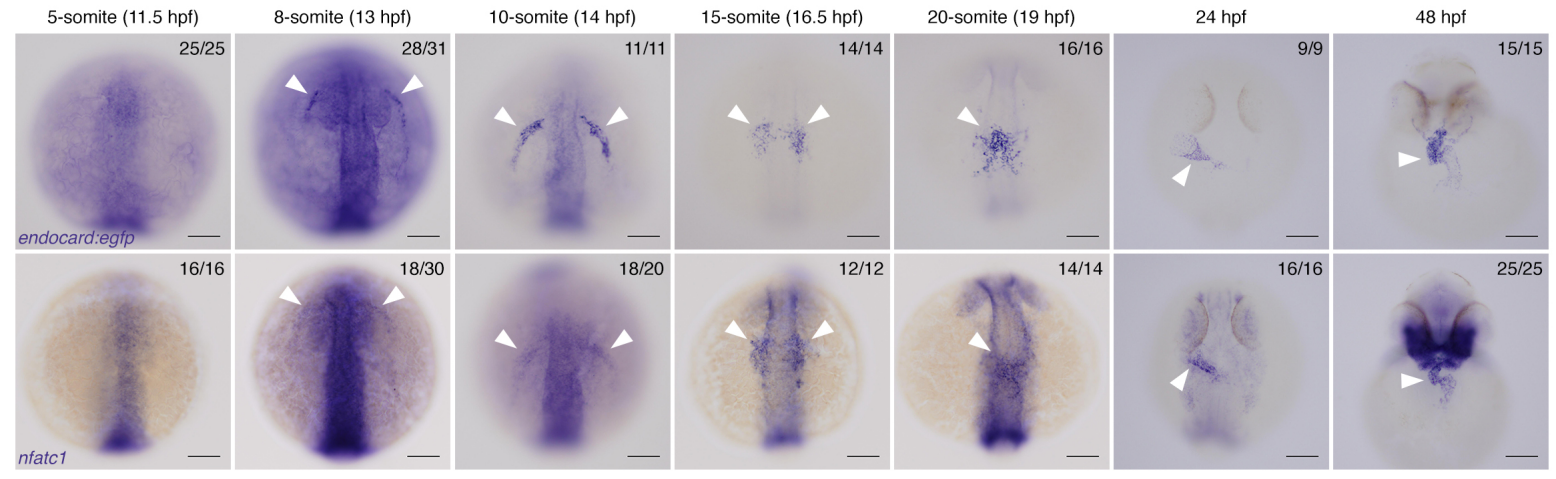

Figure 2. Restricted endocardial markers are first expressed at the 8ss in zebrafish embryos. 


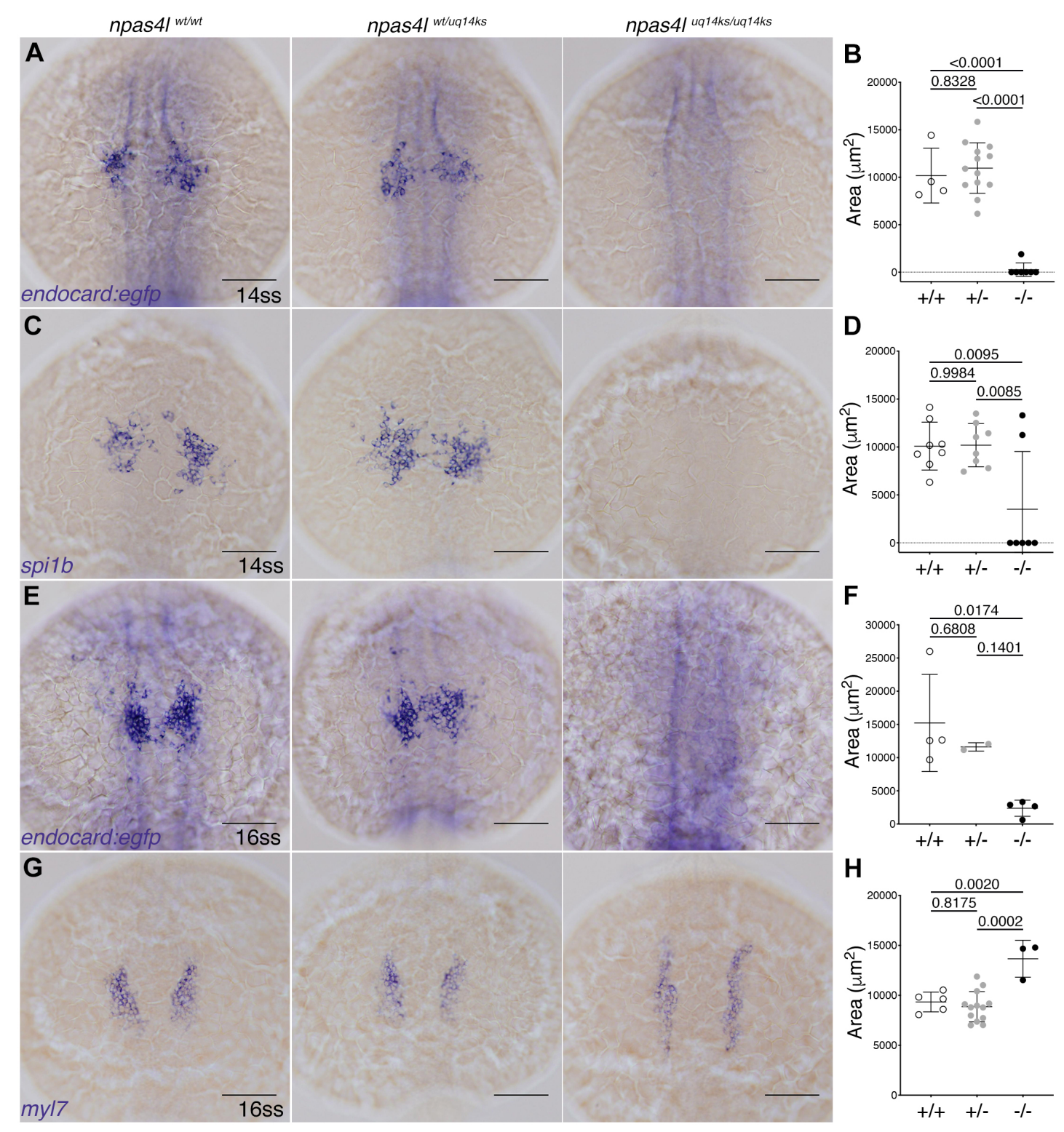

Figure 3. Gt(endocard:egfp) expression is lost in cloche/npas4l mutants. 


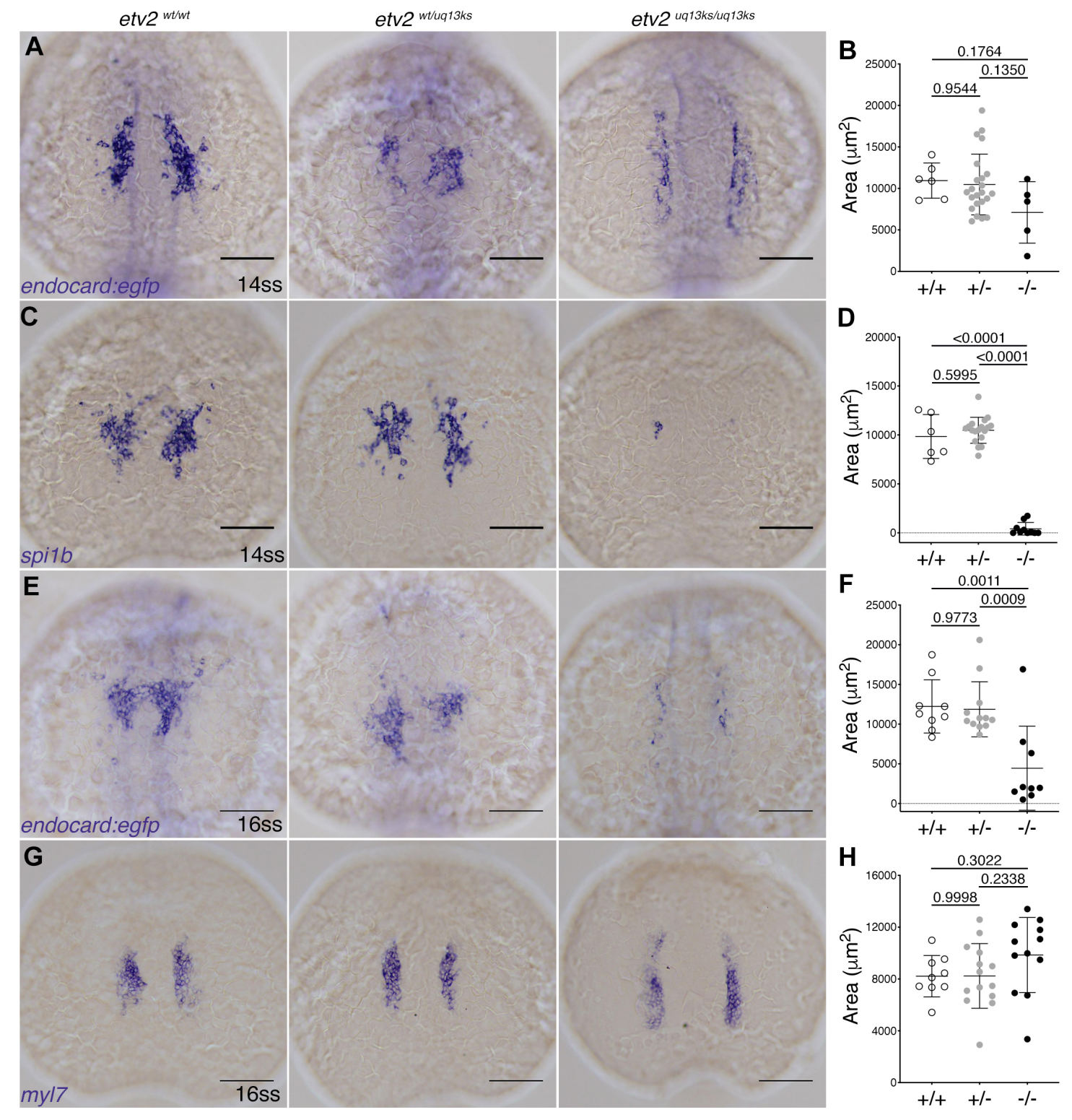

Figure 4. Gt(endocard:egfp) expression is reduced in etv2 mutants. 

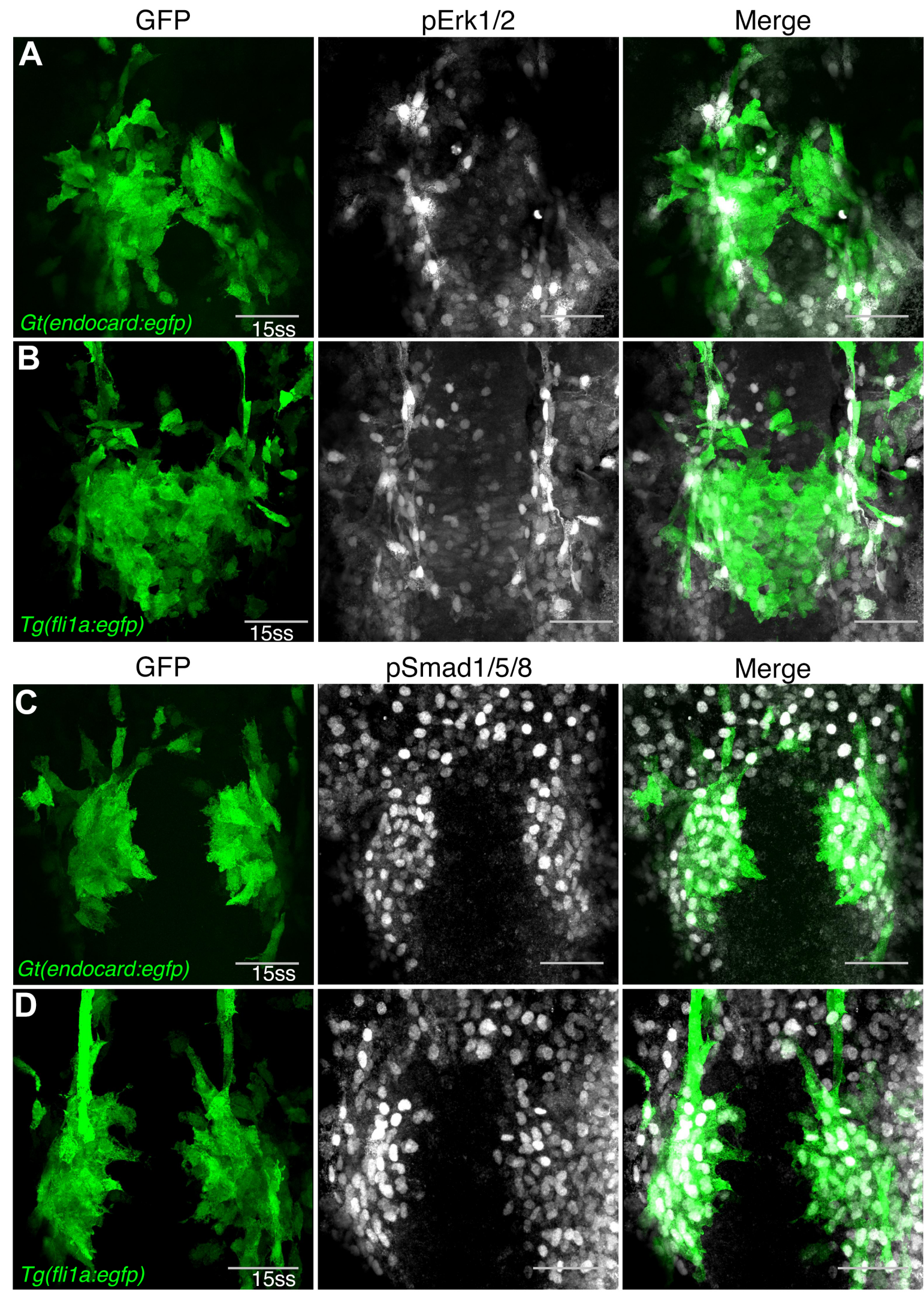

Figure 5. BMP is actively signalling in developing endocardial cells. 
bioRxiv preprint doi: https://doi.org/10.1101/654525; this version posted May 30, 2019. The copyright holder for this preprint (which was not certified by peer review) is the author/funder, who has granted bioRxiv a license to display the preprint in perpetuity. It is made available under aCC-BY 4.0 International license.
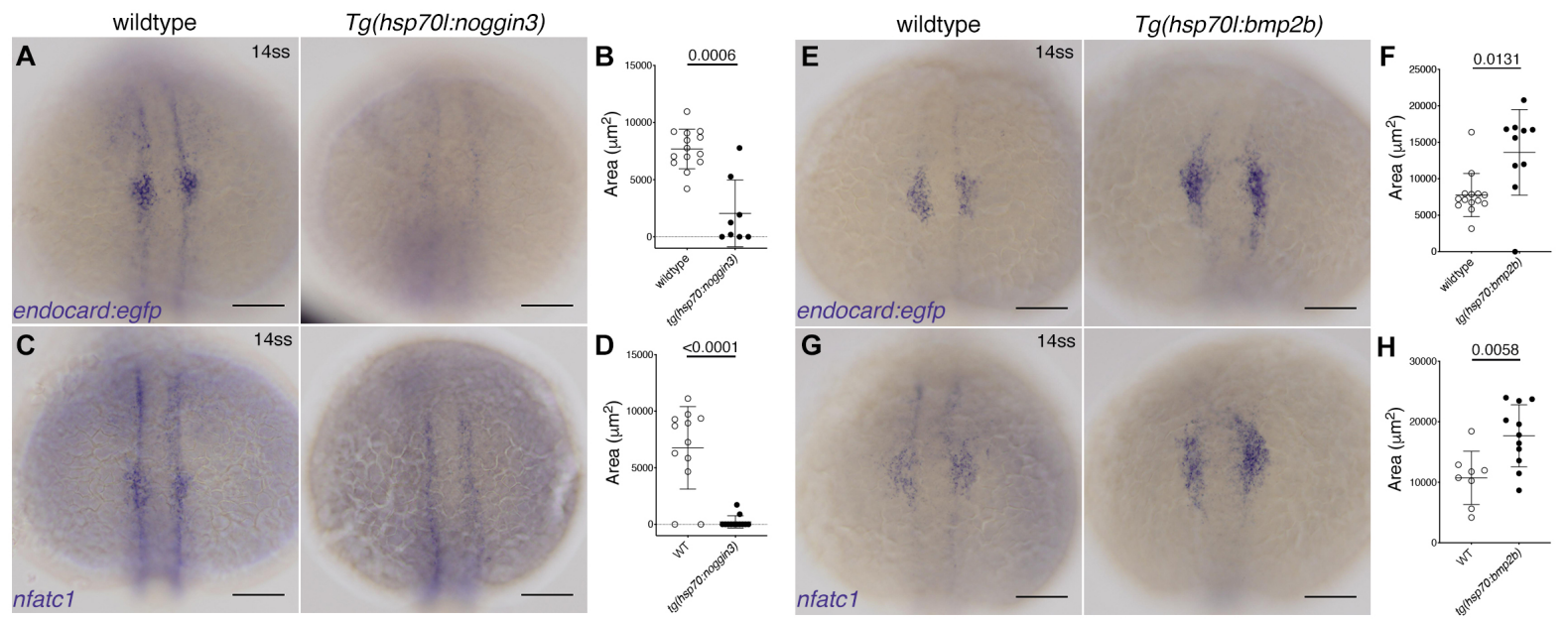

Figure 6. BMP signalling is required for endocardial development. 

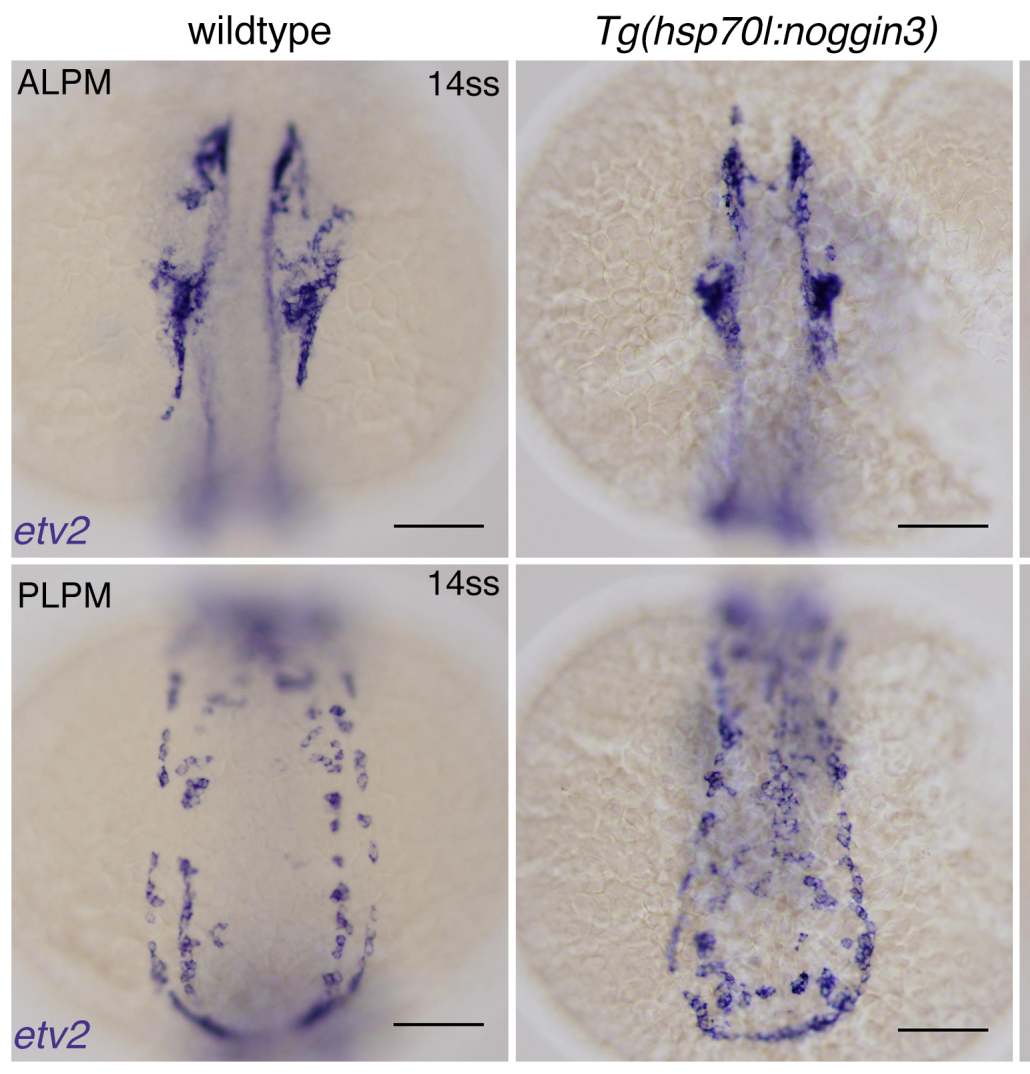

Tg(hsp70l:bmb2b)

Figure 7. etv2 expression is altered by BMP signaling modulation. 


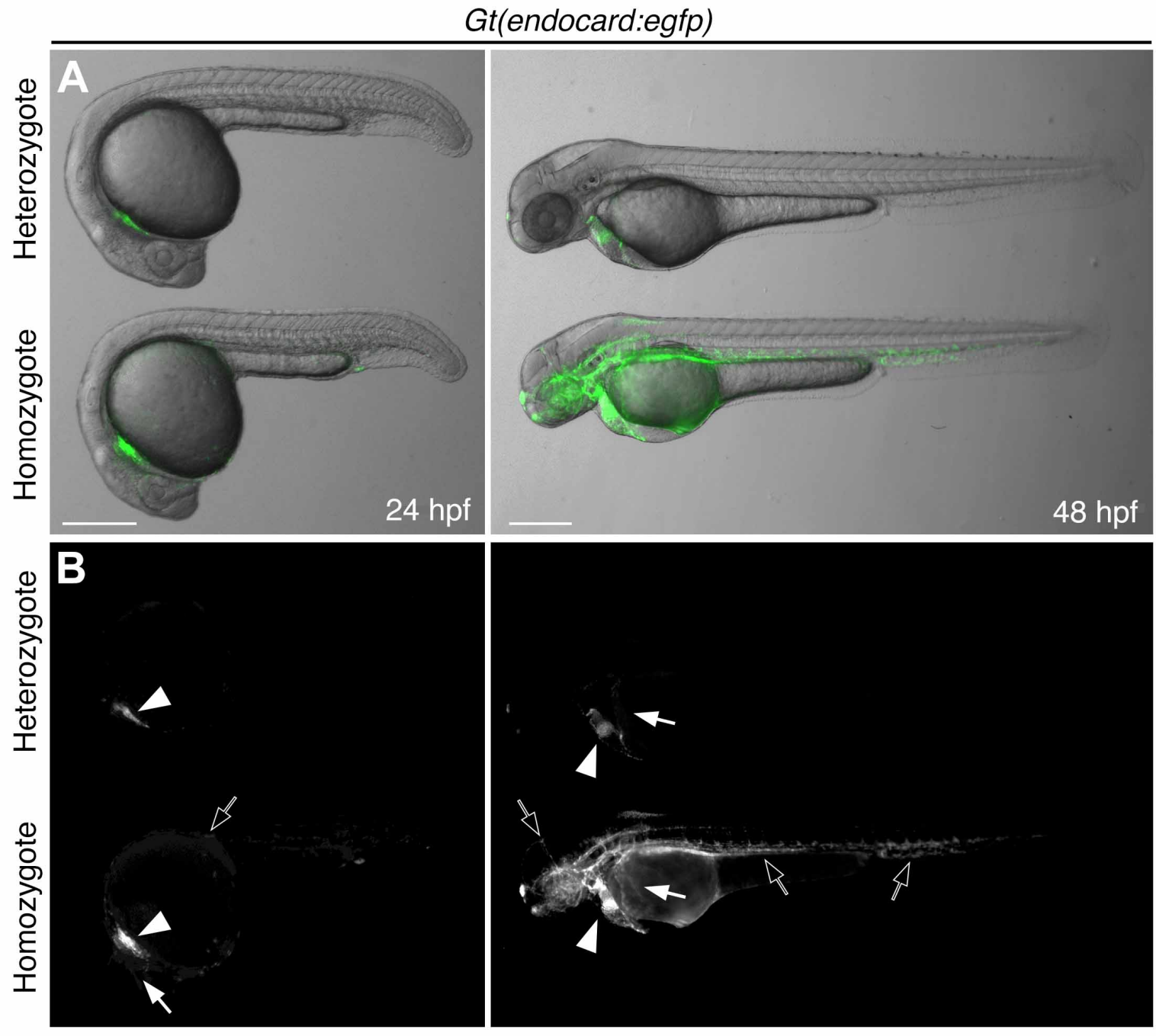

Figure 1 supplement 1. 


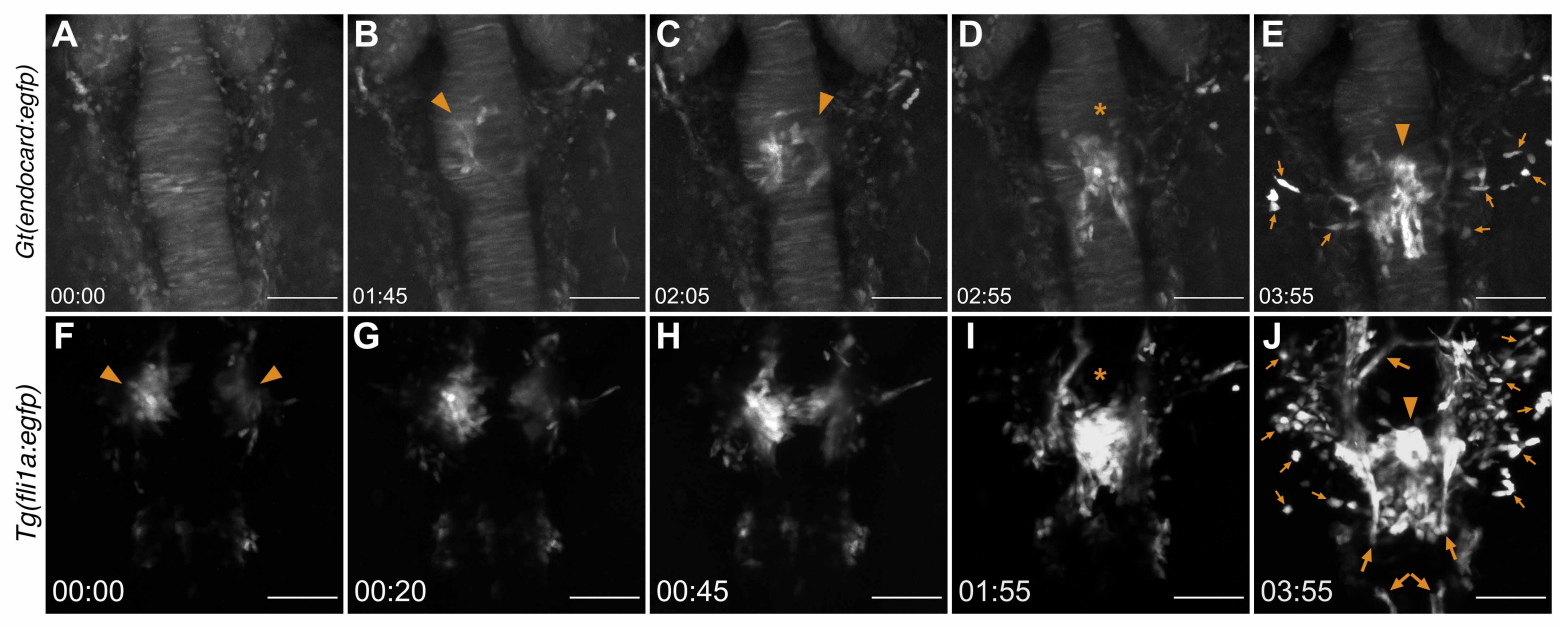

Figure 1 supplement 4 . 
bioRxiv preprint doi: https://doi.org/10.1101/654525; this version posted May 30, 2019. The copyright holder for this preprint (which was

not certified by peer review) is the author/funder, who has granted bioRxiv a license to display the preprint in perpetuity. It is made available under aCC-BY 4.0 International license.

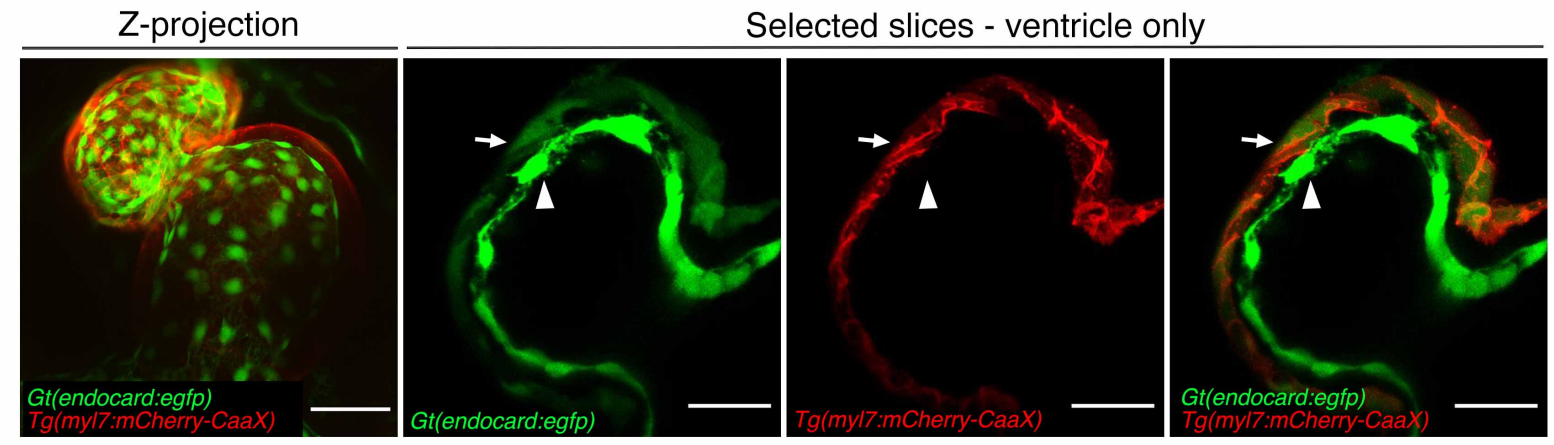

Figure 1 supplement 5. 
bioRxiv preprint doi: https://doi.org/10.1101/654525; this version posted May 30, 2019. The copyright holder for this preprint (which was

not certified by peer review) is the author/funder, who has granted bioRxiv a license to display the preprint in perpetuity. It is made available under aCC-BY 4.0 International license.

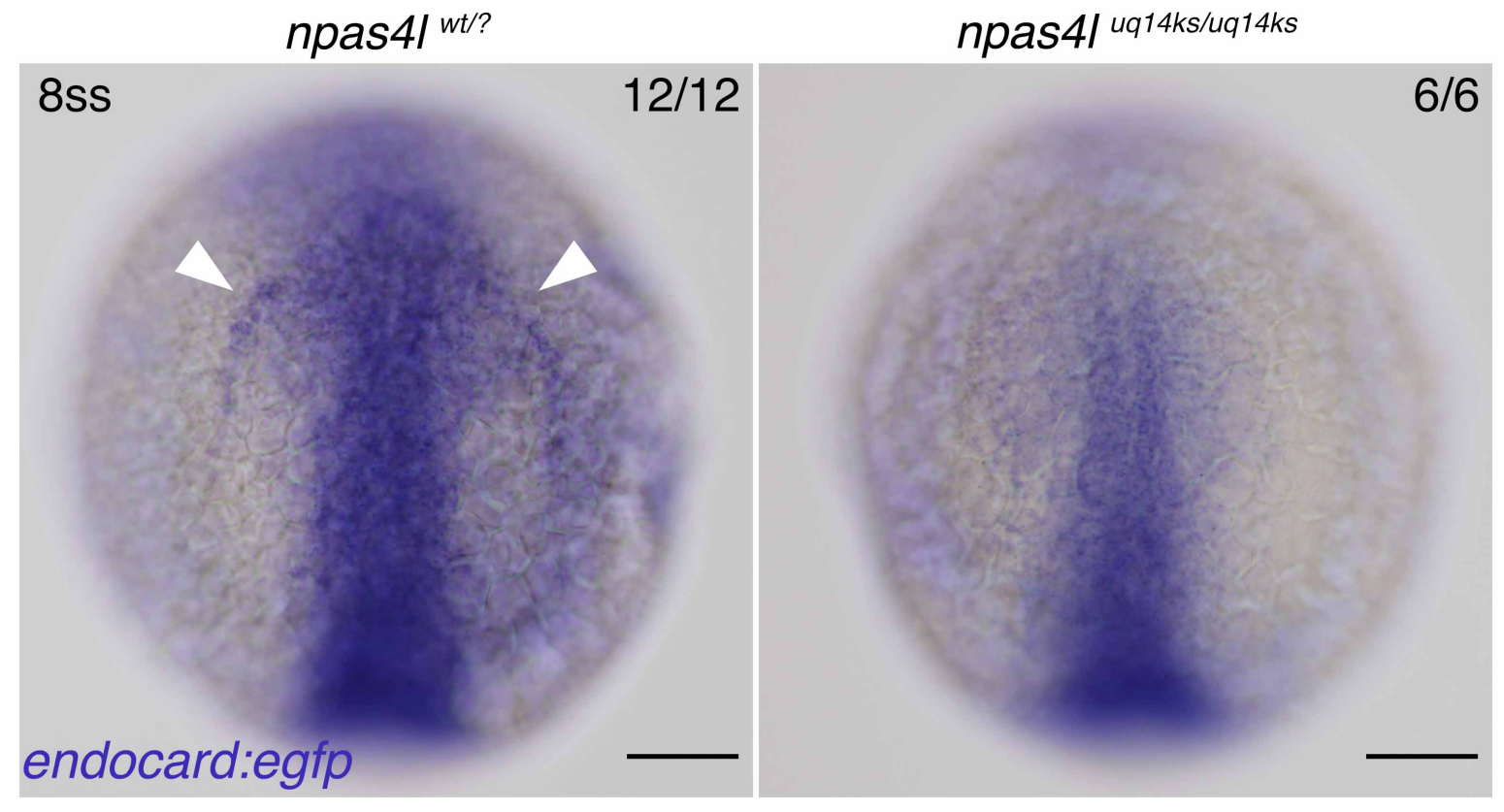

Figure 2 supplement 1. 


\section{Gt(endocard:egfp)}

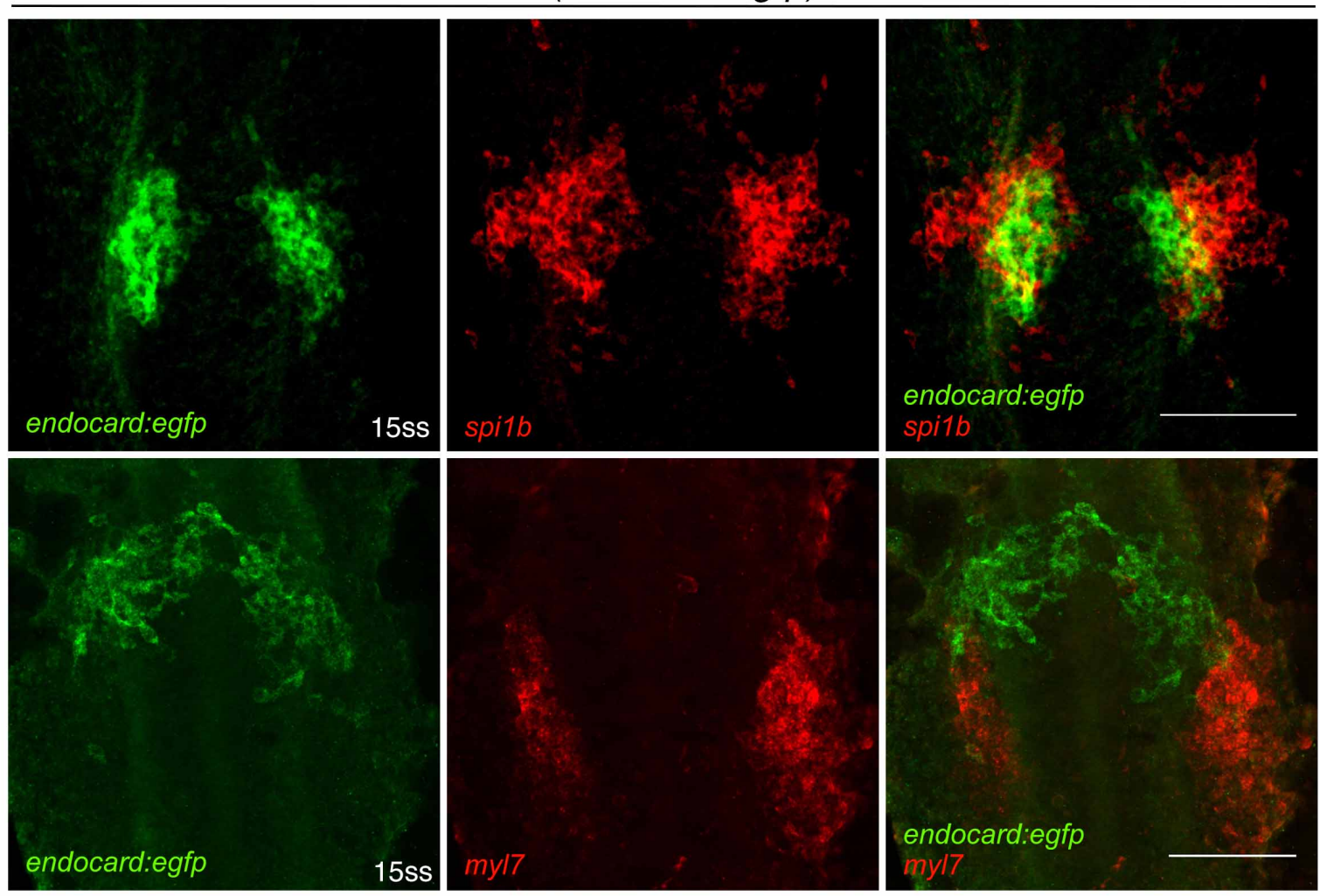

Figure 3 supplement 1. 


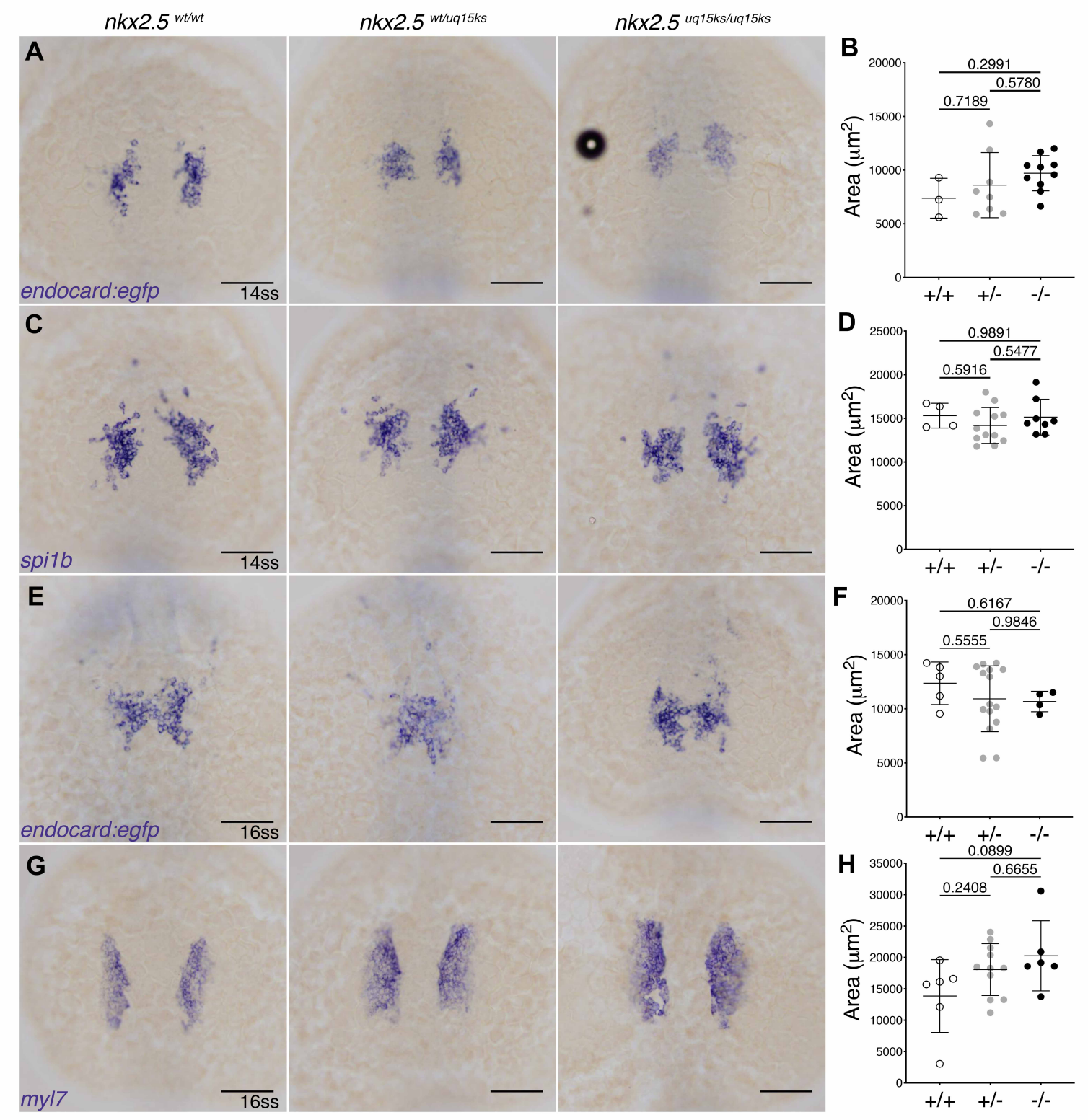

Figure 3 supplement 2. 


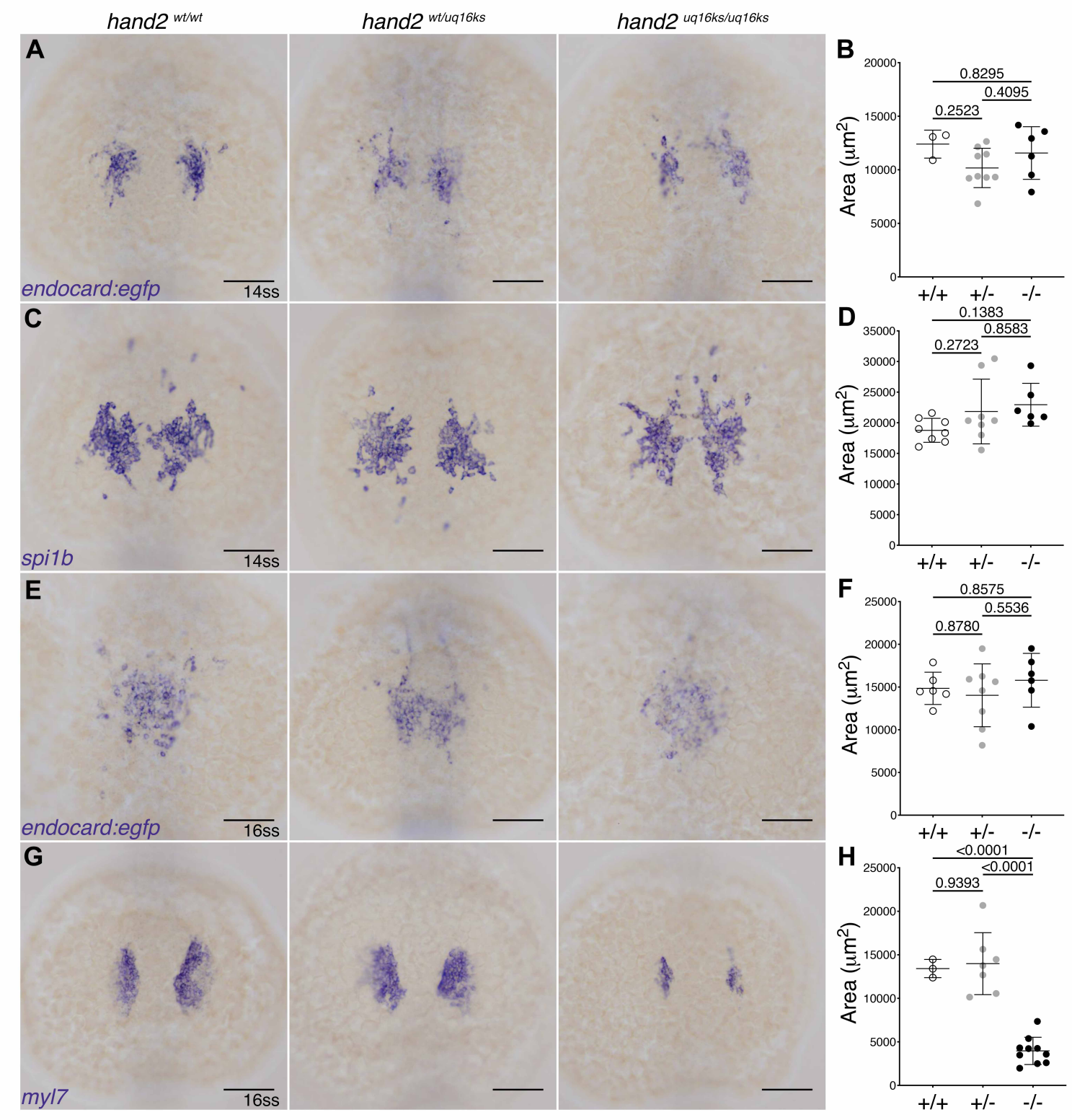

Figure 3 supplement 3. 


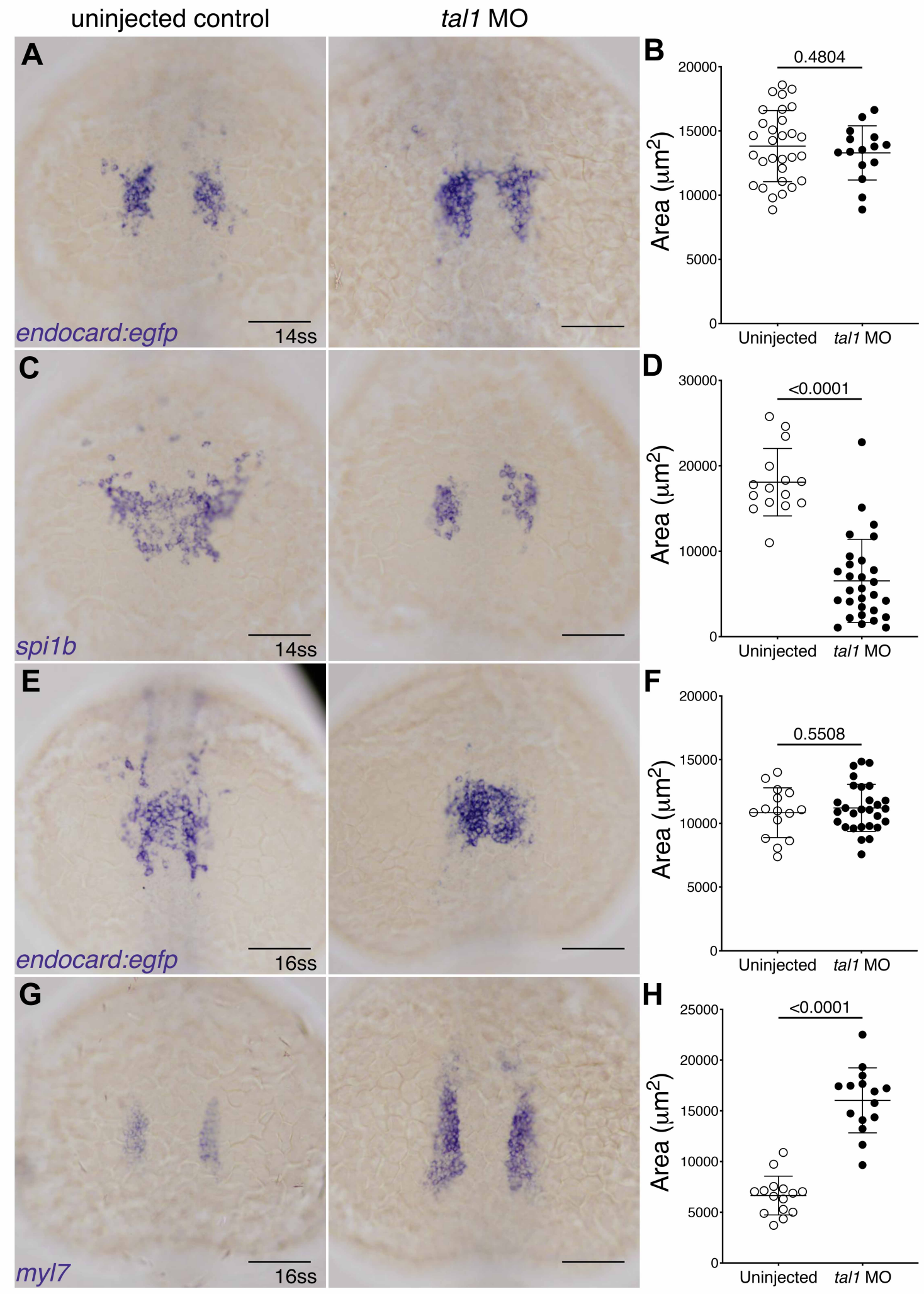

Figure 3 supplement 4. 

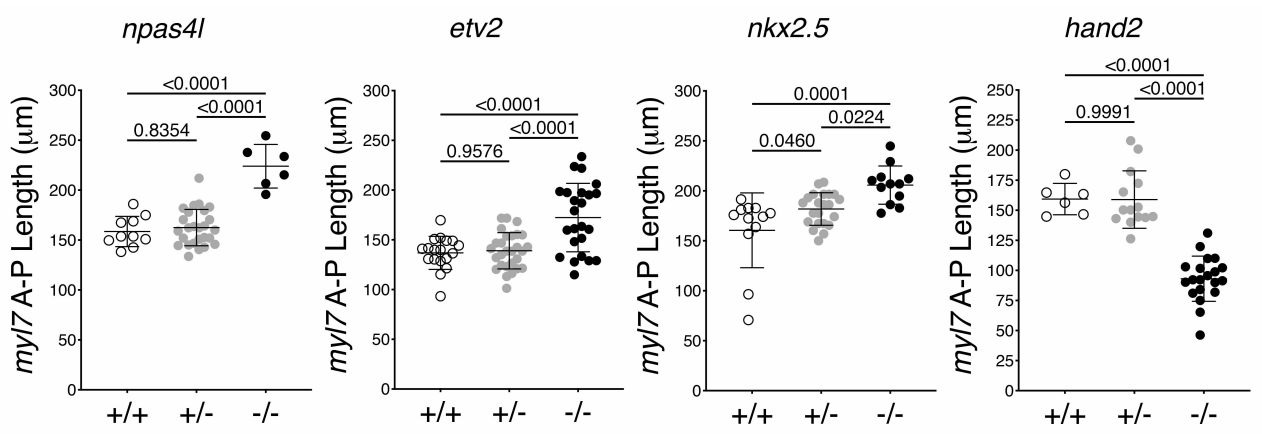

tal1 MO

Figure 3 supplement 5. 
bioRxiv preprint dol: https://doi.org/10.1101/654525; this version posted May 30, 2019. The copyright holder for this preprint (which was not certified by peer review) is the author/funder, who has granted bioRxiv a license to display the preprint in perpetuity. It is made available under aCC-BY 4.0 International license.
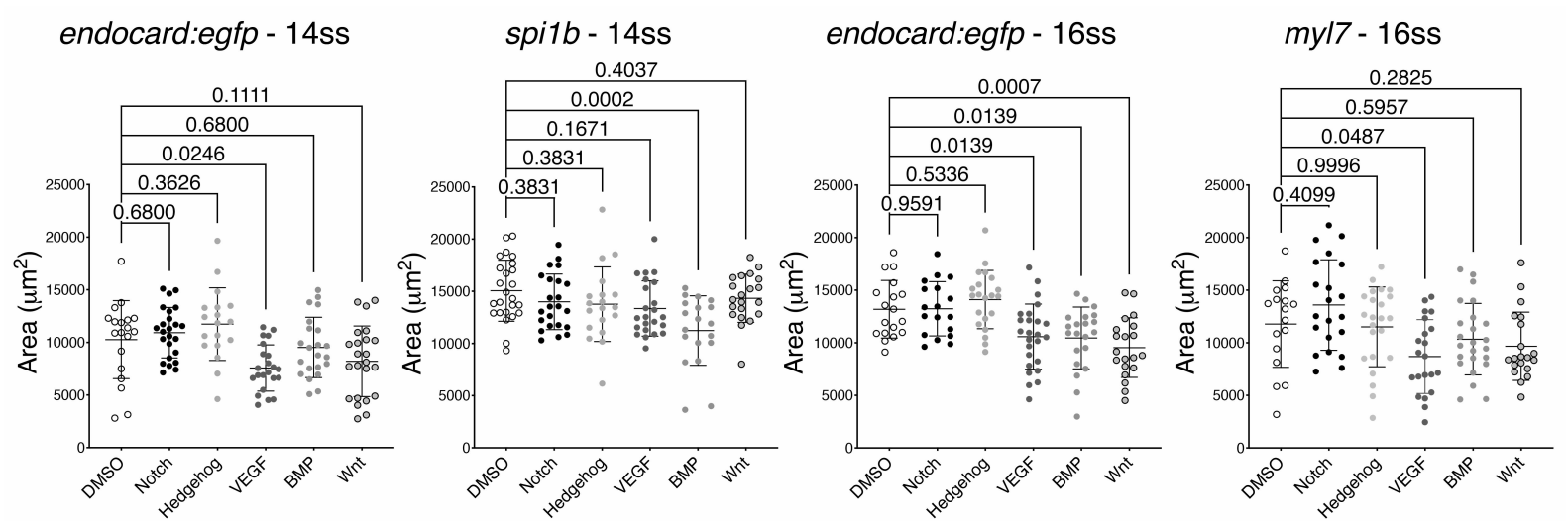

Figure 5 supplement 1. 
bioRxiv preprint doi: https://doi.org/10.1101/654525; this version posted May 30, 2019. The copyright holder for this preprint (which was

not certified by peer review) is the author/funder, who has granted bioRxiv a license to display the preprint in perpetuity. It is made available under aCC-BY 4.0 International license.
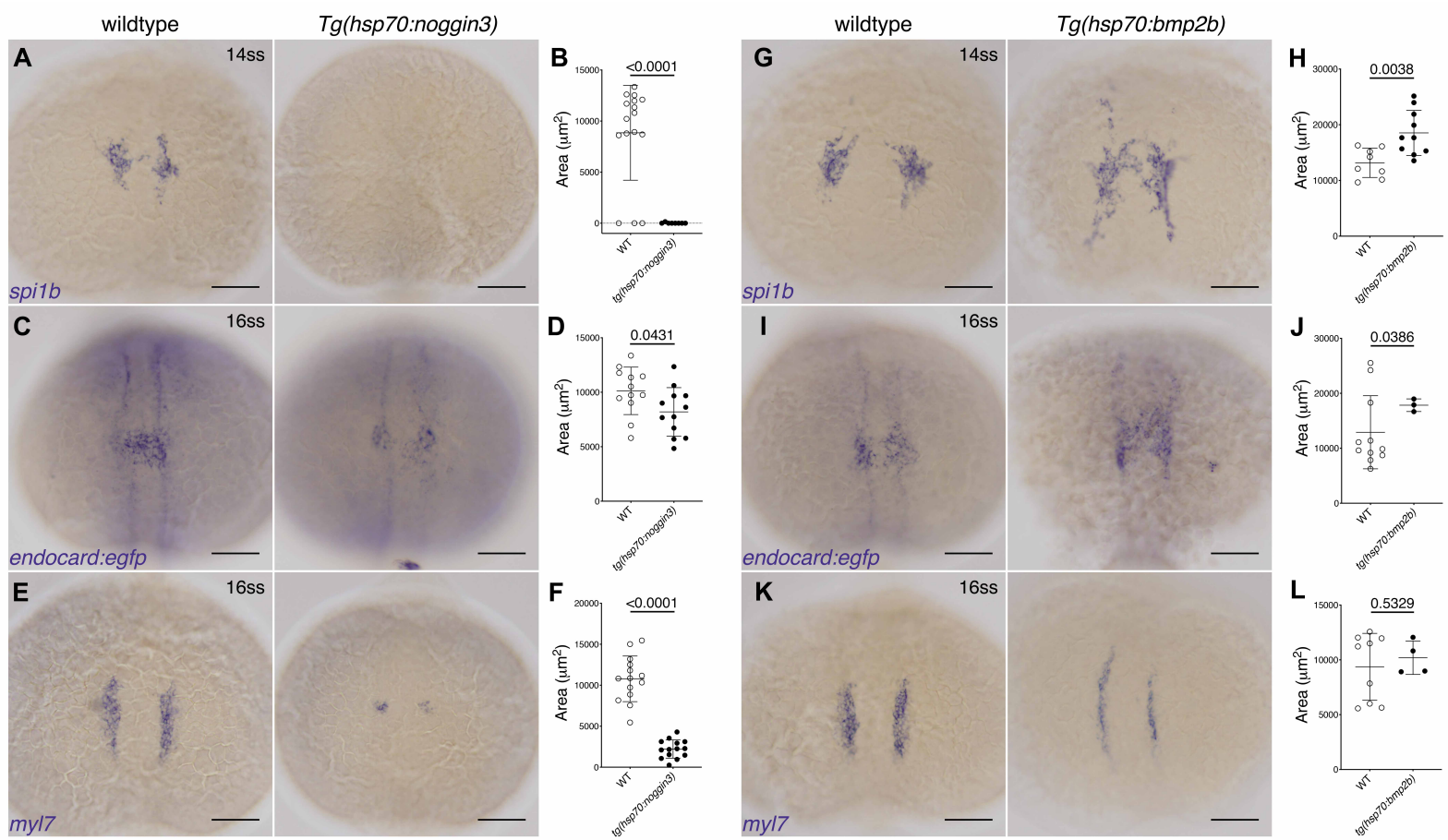

Figure 6 supplement 1. 